\title{
Mathematical Modeling of Multienzyme Biosensor System
}

\author{
SP. Ganesan, ${ }^{1}$ K. Saravanakumar, ${ }^{2}$ and L. Rajendran ${ }^{2}$ \\ ${ }^{1}$ Department of Mathematics, Syed Ammal Engineering College, Ramanathapuram, Tamil Nadu 623502, India \\ ${ }^{2}$ Department of Mathematics, The Madura College, Madurai, Tamil Nadu 625011, India \\ Correspondence should be addressed to L. Rajendran; raj_sms@rediffmail.com
}

Received 27 March 2014; Revised 18 August 2014; Accepted 8 September 2014; Published 1 October 2014

Academic Editor: Helen McAneney

Copyright (C) 2014 SP. Ganesan et al. This is an open access article distributed under the Creative Commons Attribution License, which permits unrestricted use, distribution, and reproduction in any medium, provided the original work is properly cited.

\begin{abstract}
A mathematical model of hybrid inhibitor biosensor system is discussed. This model consists of five nonlinear partial differential equations for bisubstrate sensitive amperometric system. Simple and closed form of analytical expressions for concentration of glucose-6-phosphate (substrate), potassium dihydrogen phosphate (inhibitor), oxygen (co-substrate), glucose (product 1), and hydrogen peroxide (product 3 ) is obtained in terms of rate constant using modified Adomian decomposition method (MADM). In this study, behavior of biokinetic parameters is analyzed using this theoretical result. The obtained analytical results (concentrations) are compared with the numerical results and are found to be in satisfactory agreement.
\end{abstract}

\section{Introduction}

A biosensor is an analytical device, used for the detection of an analyte or substrate that combines a biological component with a physicochemical detector [1]. Biosensors are potentially useful as they detect components quickly and have been active in the research area for certain years. The development of these biosensing systems depends on a quantitative measurement of the enzyme activity before and after exposure to a target analyte. Inhibition-based biosensors have been the topic of several recent reviews [2,3]. Biosensors based on the principle of enzyme inhibition have been applied for a wide range of significant analytes. Some recent approaches and developments in enzyme inhibition-based biosensors for food safety and environmental monitoring have been discussed by Amine et al. [4]. The application of whole-cell-based biosensors to different environmental media, such as water, soil, and atmospheric monitoring has been reviewed by $\mathrm{Gu}$ et al. [5]. An algal biosensor for toxicity assessment of estuarine waters was tested at different concentrations [6]. On biosensor technology, initial studies for the construction of a diffusive sampling device was described for monitoring personal exposure to formic acid [7]. The determination of benzene in workplace air samples using a bacterial biosensor based on flow injection analysis (FIA) has been developed by Lanyon et al. [8]. Kok and
Hasirci [9] reported that the total anticholinesterase activity of binary pesticide mixtures was lower than the sum of the individual inhibition values. Mazzei et al. [10] immobilized acid phosphatase and glucoseoxidase on a separate dialytic membrane. The bienzyme modified membrane was placed on platinum electrode using an "O" ring. On enzymatic biosensor, the steady state analytical expression of concentration of substrate is derived using variational iteration method [11]. A monolayer potentiometric biosensor response for a polymer-modified electrode system has been discussed by Rahamathunissa et al. [12]. The biosensors can be used for biomedical applications [13] and electric circuits [14]. Some nonlinear problems in different types of biosensors are solved using various numerical methods [15] and analytical methods [16].

The multienzyme biosensor system was used for the detection of phosphorous and fluoride ions. Rangelova et al. [17] investigated the influence of concentration and reaction rate constant of inhibitor with two enzymes in the dynamic mode. In this work, we study the steady state performance of multienzyme inhibitor system performing glucose-6phosphate hydrolysis at different conditions. The analytical expression for concentrations and current are derived using modified Adomian decomposition method. The effect of internal mass transfer parameters and kinetic parameters of inhibitor is discussed. 


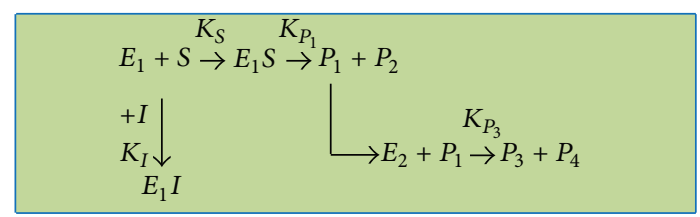

FIGURE 1: Reaction scheme of the enzyme with competitive inhibition process.

\section{Mathematical Formulation of the Problem}

In enzymatic reactions, the molecules at the beginning of the process, called substrates, are converted into different molecules, called products. Almost all chemical reactions in a biological cell need enzymes in order to occur at rates sufficient for life. The working mechanism of hybrid biosensor for phosphate is based on the following sequential biochemical reactions [8]:

$$
\begin{gathered}
\mathrm{C}_{6} \mathrm{H}_{13} \mathrm{O}_{9} \mathrm{P}+\mathrm{H}_{2} \mathrm{O} \stackrel{\mathrm{AP}}{\longrightarrow} \mathrm{C}_{6} \mathrm{H}_{12} \mathrm{O}_{6}+\mathrm{HPO}_{4}^{2-} \\
\mathrm{C}_{6} \mathrm{H}_{12} \mathrm{O}_{6}+\mathrm{O}_{2} \stackrel{\mathrm{GOD}}{\longrightarrow} \mathrm{C}_{6} \mathrm{H}_{10} \mathrm{O}_{6}+\mathrm{H}_{2} \mathrm{O}_{2}
\end{gathered}
$$

The biochemical reaction, which is biocatalyzed by acid phosphatase (AP), involves the hydrolysis of glucose-6-phosphate $\left(\mathrm{C}_{6} \mathrm{H}_{13} \mathrm{O}_{9} \mathrm{P}\right)$ with the liberation of glucose $\left(\mathrm{C}_{6} \mathrm{H}_{12} \mathrm{O}_{6}\right)$ and inorganic phosphate $\left(\mathrm{HPO}_{4}^{2-}\right)$. In the second reaction with glucose oxidase (GOD), the oxygen present oxidizes the glucose into glucono delta-lactone $\left(\mathrm{C}_{6} \mathrm{H}_{10} \mathrm{O}_{6}\right)$ and hydrogen peroxide $\left(\mathrm{H}_{2} \mathrm{O}_{2}\right)$. The amount of hydrogen peroxide being produced is measured electrochemically. On the basis of the biochemical reactions, the following successive enzyme reactions with competitive inhibition have been proposed:

$$
\begin{gathered}
S \stackrel{E_{1}}{\longrightarrow} P_{1}+P_{2}, \\
I \stackrel{E_{1}}{\longrightarrow} E_{1} I, \\
P_{1}+C \stackrel{E_{2}}{\longrightarrow} P_{3}+P_{4},
\end{gathered}
$$

where $S, I, C, P_{1}$, and $P_{3}$ are the concentrations of the glucose6-phosphate (substrate), potassium dihydrogen phosphate (inhibitor), oxygen (co-substrate), glucose (product 1), and hydrogen peroxide (product 3 ) respectively. $E_{1}$ (acid phosphatase) is the first enzyme and $E_{2}$ (glucose oxidase) is the second enzyme. $P_{2}$ and $P_{4}$ represent the inorganic phosphate and glucono delta-lactone. Figure 1 presents the overall reaction scheme for a multienzyme inhibitor system. We assume that diffusion is one-dimensional in space and is described with second Fick's law. The concentration profiles of five reagents of the system are described by the following nonlinear equations:

$$
\begin{gathered}
\frac{\partial S}{\partial t}=D_{S} \frac{\partial^{2} S}{\partial x^{2}}-\frac{V_{1} S}{K_{S}\left[1+I / K_{I}\right]+S} \\
\frac{\partial I}{\partial t}=D_{I} \frac{\partial^{2} I}{\partial x^{2}}-\frac{V_{1} S}{K_{S}\left[1+I / K_{I}\right]+S}, \\
\frac{\partial C}{\partial t}=D_{C} \frac{\partial^{2} C}{\partial x^{2}}-\frac{V_{2}}{1+K_{P_{1}} / P_{1}+K_{C} / C} \\
\frac{\partial P_{1}}{\partial t}=D_{P_{1}} \frac{\partial^{2} P_{1}}{\partial x^{2}}+\frac{V_{1} S}{K_{S}\left[1+I / K_{I}\right]+S}-\frac{V_{2}}{1+K_{P_{1}} / P_{1}+K_{C} / C} \\
\frac{\partial P_{3}}{\partial t}=D_{P_{3}} \frac{\partial^{2} P_{3}}{\partial x^{2}}+\frac{V_{2}}{1+K_{P_{1}} / P_{1}+K_{C} / C}
\end{gathered}
$$

where $D_{S}, D_{C}, D_{P_{1}}$, and $D_{P_{3}}$ are diffusion coefficients for substrate, co-substrate, product 1 , and product $3 . K_{i}$ denote the reaction constant for concentration profiles $\left(i=S, I, C, P_{1}\right)$, respectively. $V_{1}$ is the reaction velocity of acid phosphatase and $V_{2}$ is the reaction velocity of glucose oxidase. The initial conditions are

$$
\begin{gathered}
t=0, \quad S(x, 0)=S_{0} ; \quad I(x, 0)=I_{0} ; \\
C(x, 0)=C_{0} ; \quad P_{1}(x, 0)=0 ; \quad P_{3}(x, 0)=0 .
\end{gathered}
$$

Equations (4) are subjected to the following boundary conditions:

$$
\begin{gathered}
x=0, \quad S(0, t)=S_{0} ; \quad I(0, t)=I_{0} ; \\
C(0, t)=C_{0} ; \quad P_{1}(0, t)=0 ; \quad P_{3}(0, t)=0, \\
x=d, \quad \frac{\partial S}{\partial x}=0 ; \quad I(d, t)=0 ; \\
C(d, t)=0 ; \quad \frac{\partial P_{1}}{\partial x}=0 ; \quad P_{3}(d, t)=0 .
\end{gathered}
$$

The output current is defined as

$$
i=-\left.n F A D_{P_{3}} \frac{\partial P_{3}}{\partial x}\right|_{x=d}
$$

where $n$ is the number of electrons, $F$ is the Faraday's constant, and $A$ is the area of the electrode surface. 


\section{Governing Equations in Dimensionless Form}

Equations (4)-(7) can be reduced to the corresponding dimensionless forms by introducing the following dimensionless parameters:

$$
\begin{gathered}
u=\frac{S}{S_{0}}, \quad v=\frac{I}{I_{0}}, \quad w=\frac{C}{C_{0}}, \\
a=\frac{P_{1}}{S_{0}}, \quad b=\frac{P_{3}}{C_{0}}, \quad \tau=\frac{D_{S} t}{d^{2}}, \\
X=\frac{x}{d}, \quad \alpha=\frac{I_{0}}{K_{I}}, \quad \beta=\frac{S_{0}}{K_{S}}, \\
\theta=\frac{K_{C}}{C_{0}}, \quad \gamma=\frac{K_{P_{1}}}{S_{0}}, \quad \phi_{U}^{2}=\frac{V_{1} d^{2}}{D_{S} K_{S}}, \\
\psi_{V}^{2}=\frac{V_{2} d^{2}}{D_{I} I_{0}}, \quad \psi_{W}^{2}=\frac{V_{2} d^{2}}{D_{C} C_{0}}, \quad \phi_{A}^{2}=\frac{V_{1} d^{2}}{D_{P_{1}} K_{S}}, \\
\psi_{A}^{2}=\frac{V_{2} d^{2}}{D_{P_{1}} S_{0}}, \quad \psi_{B}^{2}=\frac{V_{2} d^{2}}{D_{P_{3}} C_{0}} .
\end{gathered}
$$

Consequently, (4)-(7) can be written in dimensionless form for steady state as follows:

$$
\begin{gathered}
\frac{d^{2} u}{d X^{2}}=\frac{\phi_{U}^{2} u}{1+\alpha v+\beta u} \\
\frac{d^{2} v}{d X^{2}}=\frac{\psi_{V}^{2} u}{1+\alpha v+\beta u} \\
\frac{d^{2} w}{d X^{2}}=\frac{\psi_{W}^{2}}{1+\gamma / a+\theta / w} \\
\frac{d^{2} a}{d X^{2}}=\frac{\psi_{A}^{2}}{1+\gamma / a+\theta / w}-\frac{\phi_{A}^{2} u}{1+\alpha v+\beta u} \\
\frac{d^{2} b}{d X^{2}}=-\frac{\psi_{B}^{2}}{1+\gamma / a+\theta / w}
\end{gathered}
$$

The boundary conditions are

$$
\begin{gathered}
X=0, \quad u=1 ; \quad v=1 ; \\
w=1 ; \quad a=\varepsilon ; \quad b=0, \\
X=1, \quad \frac{d u}{d X}=1 ; \quad v=0 ; \\
w=0 ; \quad \frac{d a}{d X}=0 ; \quad b=0 .
\end{gathered}
$$

Here we can assume that $\varepsilon$ is very small $(\varepsilon=0)$ at $X=0$.

The dimensionless current is as follows:

$$
I=\frac{I d}{n F A D_{P_{3}} c_{0}}=-\left.\frac{d b}{d X}\right|_{X=1} .
$$

\section{Analytical Expressions of the \\ Concentrations and Current Using Modified Adomian Decomposition Method}

In order to solve the boundary value problem, (9)-(13), we used the modified Adomian decomposition method [18, 19]. The basic idea of this method is described in Appendix A [20]. Detailed derivations of the dimensionless concentrations $u, v, w, a$, and $b$ of glucose-6-phosphate, potassium dihydrogen phosphate, oxygen, glucose, and hydrogen peroxide are described in Appendix B. As a result, we have obtained

$$
\begin{aligned}
u(X)= & 1-\frac{\phi_{U}^{2} X}{c}\left[\log \left(\frac{c+d}{d}\right)+1\right] \\
& +\frac{\phi_{U}^{2}}{c}\left[\frac{c X+d}{c}\right] \log \left(\frac{c X+d}{d}\right), \\
v(X)= & 1-X-\frac{\psi_{V}^{2} \beta X}{c}\left[\frac{c+d}{c}\right] \log \left(\frac{c+d}{d}\right) \\
& +\frac{\psi_{V}^{2} \beta}{c}\left[\frac{c X+d}{c}\right] \log \left(\frac{c X+d}{d}\right),
\end{aligned}
$$

$$
\begin{aligned}
w(X)=1- & X+\frac{\varepsilon \psi_{W}^{2}}{p^{3}} \\
\times & \left\{(p X+q)\left[p \log \left(\frac{p X+q}{q}\right)+q \log (p X+q)\right]\right. \\
& +\frac{1-X}{2}\left[p^{2} X-2 q^{2} \log (q)\right] \\
& \left.\quad-(p+q) X\left[p \log \left(\frac{p+q}{q}\right)+q \log (p+q)\right]\right\},
\end{aligned}
$$

$$
a(X)=\varepsilon+\frac{\phi_{A}^{2} X}{c}\left[\log \left(\frac{c+d}{d}\right)+1\right]
$$

$$
\begin{aligned}
& -\left[\frac{\phi_{A}}{c}\right]^{2}(c X+d) \log \left(\frac{c X+d}{d}\right)-\frac{\varepsilon \psi_{A}^{2}}{p^{3}} \\
& \times\left\{b^{2} \log (q)-(p X+q)\right.
\end{aligned}
$$$$
\times\left[p \log \left(\frac{p X+q}{q}\right)+q \log (p X+q)\right]
$$

$$
+p X\left[p \log \left(\frac{p+q}{q}\right)+q \log (p+q)\right]
$$$$
\left.+\frac{a X}{2}(a X+2 b)\right\}
$$ 


$$
\begin{aligned}
b(X)= & -\frac{\varepsilon \psi_{B}^{2}}{p^{3}} \\
& \times\left\{(p X+q)\left[p \log \left(\frac{p X+q}{q}\right)+q \log (p X+q)\right]\right. \\
& +\frac{1-X}{2}\left[p^{2} X-2 q^{2} \log (q)\right] \\
& \left.-(p+q) X\left[p \log \left(\frac{p+q}{q}\right)+q \log (p+q)\right]\right\},
\end{aligned}
$$

where

$$
\begin{gathered}
p=-(\varepsilon+\gamma), \quad q=\gamma+\varepsilon(1+\theta), \\
c=-\alpha, \quad d=1+\alpha+\beta .
\end{gathered}
$$

Equations (16)-(20) represent the analytical expression of the concentration profiles of five reagents for all values of the dimensionless parameters $\alpha, \beta, \theta, \gamma, \phi$ and $\psi$. Also, these expressions satisfy the boundary conditions (14). The current response is given by

$$
\begin{aligned}
I= & -\frac{\varepsilon \psi_{B}^{2}}{p^{3}} \\
& \times\left\{\frac{p}{2}(p+2 q)+q^{2} \log (q)\right. \\
& \left.-q\left[p \log \left(\frac{p+q}{q}\right)+q \log (p+q)\right]\right\} .
\end{aligned}
$$

\section{Numerical Simulation}

The nonlinear differential equations (9)-(13) with boundary conditions (14) have been solved numerically using SCILAB/MATLAB software. A respective script pdex4 is provided in Algorithm 1. The numerical solution is compared with our analytical results in Figures 2-6. The comparison reveals that the analytical dimensionless concentrations and numerical results are in satisfactory agreement. The dimensionless concentration of substrate and product 3 obtained using (16) and (20) are reported in Tables 1 and 2. In these tables, our result is compared with simulation results. The average relative error between our analytical result for the concentration of substrate and simulation result are $1.487 \%$ when $\beta=25$ and $0.00 \%$ when $\beta=50,100$ and 250 (Table 1) whereas the average relative error in the case for concentration of product 3 is $0.00 \%$ for all values of $\psi_{B}$ (Table 2).

\section{Results and Discussion}

The normalized nonlinear differential equations were solved using modified Adomian decomposition method. Equations (16)-(20) represent the analytical expressions for the dimensionless concentrations of glucose-6-phosphate $u(X)$, potassium dihydrogen phosphate $v(X)$, oxygen $w(X)$, glucose
$a(X)$, and hydrogen peroxide $b(X)$. Figure 2 demonstrates quantitatively the effects of dimensionless substrate inhibition modulus, dimensionless substrate Thiele modulus, and dimensionless inhibitor inhibition modulus on the dimensionless concentration profile of substrate $u(X)$ as a function of dimensionless distance $X$ using (16). It shows that the value of $u(X)$ approaches unity when $X=0$ for all values of $\beta, \phi_{U}$, and $\alpha$. It is evident that the substrate concentration $u(X)$ increases as a result of increasing dimensionless inhibition modulus $\alpha$ and dimensionless substrate modulus $\beta$. Further, the substrate concentration $u(X)$ decreases when dimensionless Thiele modulus $\phi_{U}$ or thickness of the membrane increases.

Figure 3 represents the dimensionless concentration profile of the inhibitor $v(X)$ versus dimensionless distance $X$ using (17). It is clear that for the increasing values of dimensionless inhibitor Thiele modulus $\psi_{V}$ and dimensionless substrate inhibition modulus $\beta$, the concentration profile $v(X)$ deviates from the linear form. Also, the inhibitor concentration $v(X)$ increases when dimensionless inhibitor inhibition modulus $\alpha$ increases (see Figure 3(f)). The dimensionless concentration profile of co-substrate $w(X)$ against dimensionless distance $X$ is plotted in Figure 4. From these Figures, we conclude that the value of $w(X)$ decreases when the dimensionless co-substrate Thiele modulus $\psi_{W}$ increases. Furthermore, the co-substrate profile $w(X)$ increases when dimensionless Michaelis-Menten constant of glucose $\gamma$ and oxygen $\theta$ increases (see Figures 4(e) and 4(f)).

From Figure 5, it is clear that the dimensionless concentration profile of product $1 a(X)$ increases when product 1 dimensionless Thiele modulus $\phi_{A}$ or thickness of the membrane increases. It also decreases with decreasing $X$, approaching zero at $X=0$. It shows that the value of $a(X)$ decreases as a result of increasing dimensionless inhibition modulus $\alpha$ and dimensionless substrate inhibition modulus $\beta$. Figure 6 illustrates the dimensionless concentration profile of the product $3 b(X)$ versus dimensionless distance $X$ using (20). From these Figures, it is inferred that the product 3 concentration $b(X)$ is in the form of parabola with $X=0.5$ as the axis of parabola. The value of the concentration of product increases when dimensionless Michaelis-Menten constants $\gamma$ and $\theta$ decrease. It also shows that the value of $b(X)$ decreases when product 3 dimensionless Thiele modulus $\psi_{B}$ decreases.

Figures 7(a)-7(b) show the effect of dimensionless Michaelis-Menten constant $\gamma$ on the dimensionless current as a function of product 3 dimensionless Thiele modulus $\psi_{B}$ with $\theta$. The dimensionless current $I$ approaches zero and it is independent of $\gamma$ at low dimensionless Thiele modulus. Also, the dimensionless current increases with decreasing $\gamma$ at high dimensionless Thiele modulus. Figures 7(c)-7(d) represent the effect of Thiele modulus $\psi_{B}$ on the dimensionless current as a function of product 3 dimensionless Michaelis-Menten constant $\gamma$ with $\theta$. The dimensionless current $I$ decreases with decreasing dimensionless Thiele modulus $\psi_{B}$. From the Figures 7(e)-7(f), it is inferred that the dimensionless current $I$ increases when dimensionless Michaelis-Menten constant of glucose $\gamma$ decreases for some fixed values of $\psi_{B}$. 


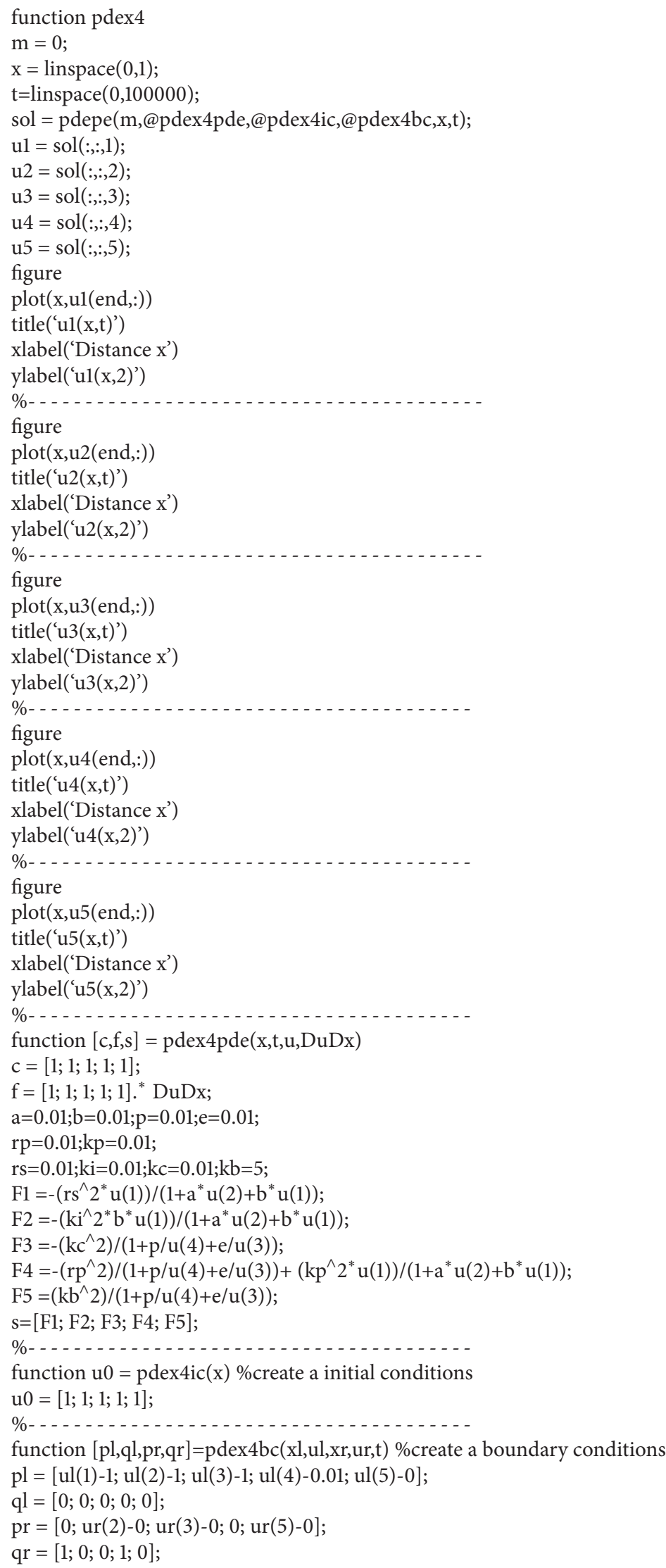




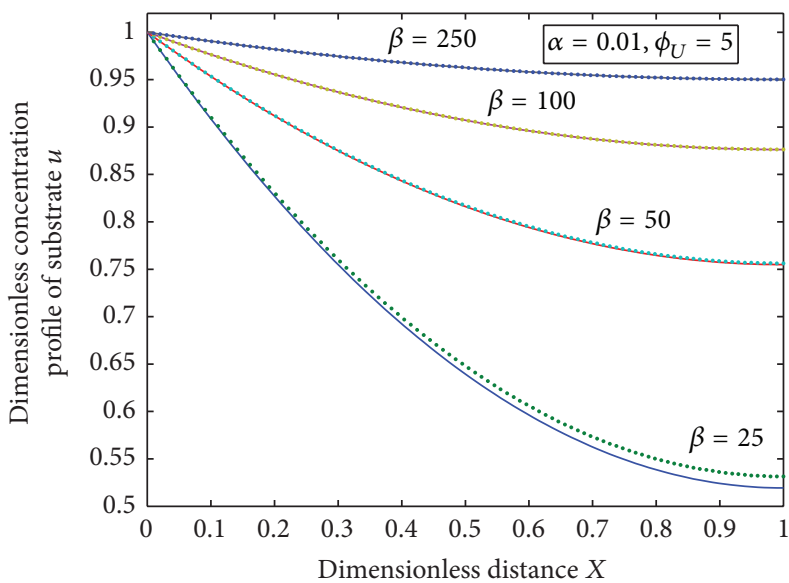

(a)

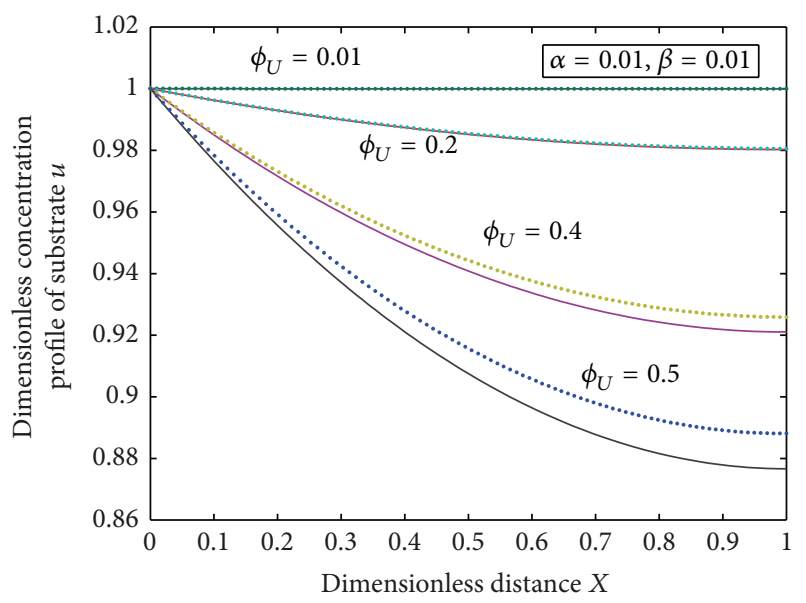

(c)

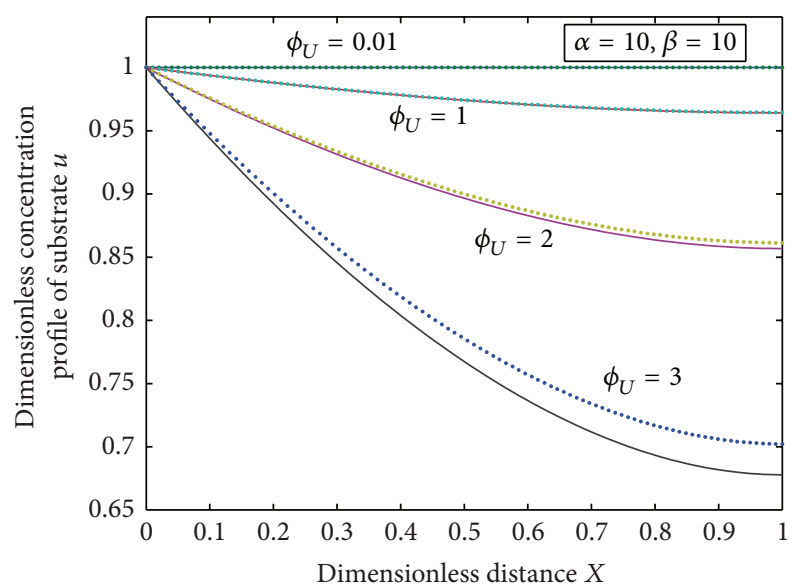

(e)

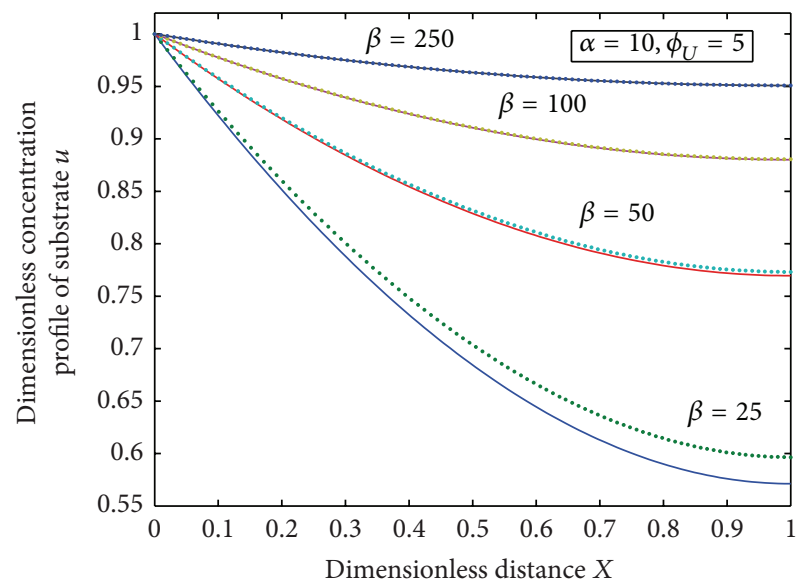

(b)

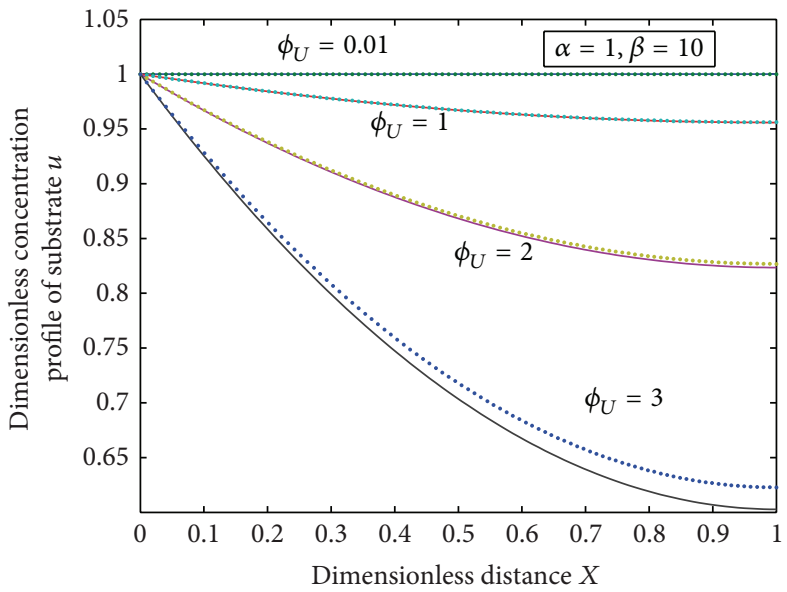

(d)

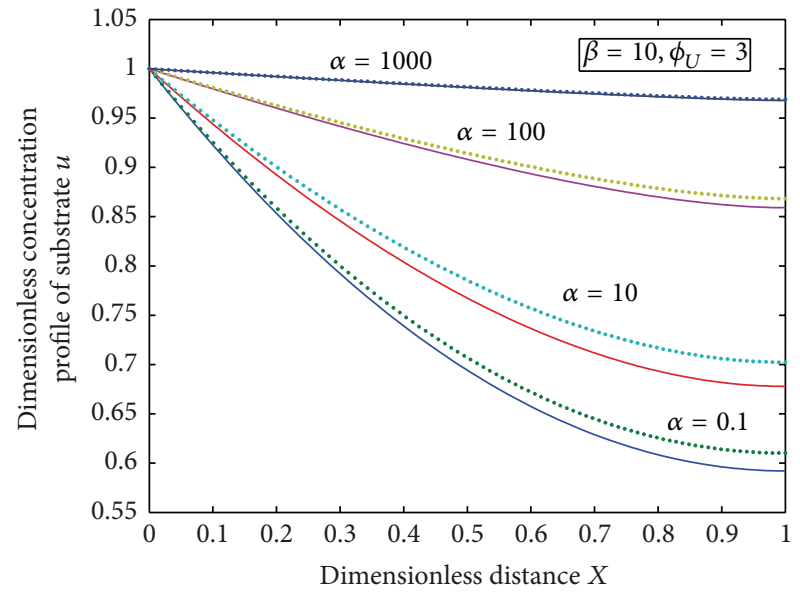

(f)

Figure 2: Plot of dimensionless substrate concentration $u$ versus dimensionless distance $X$ for various values of $\alpha, \beta$, and $\phi_{U}$. The key to the graph (solid line) represents our approximate solution presented in this study (see (16)) and (dotted line) represents the numerical solution. 


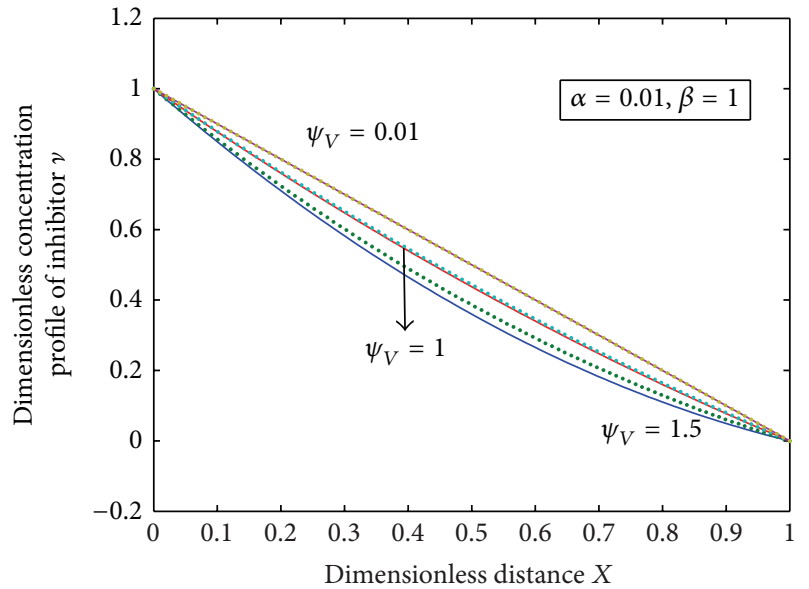

(a)

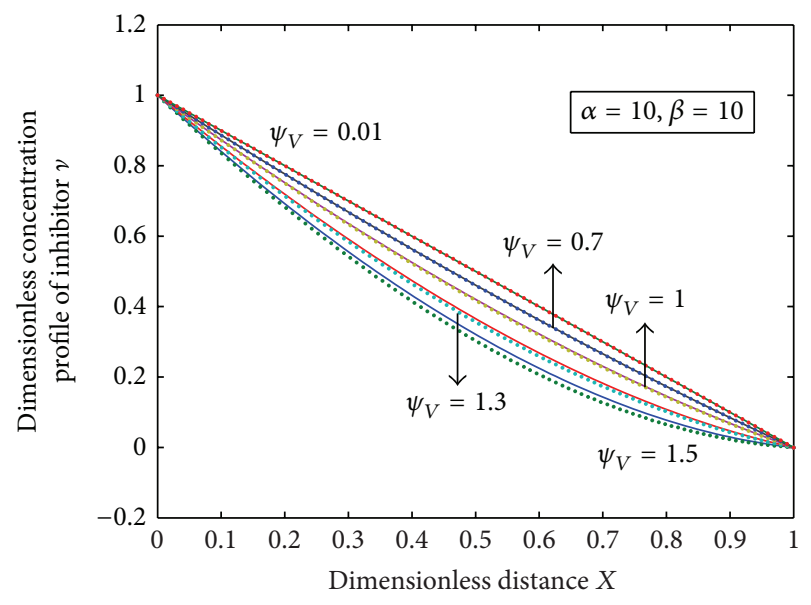

(c)

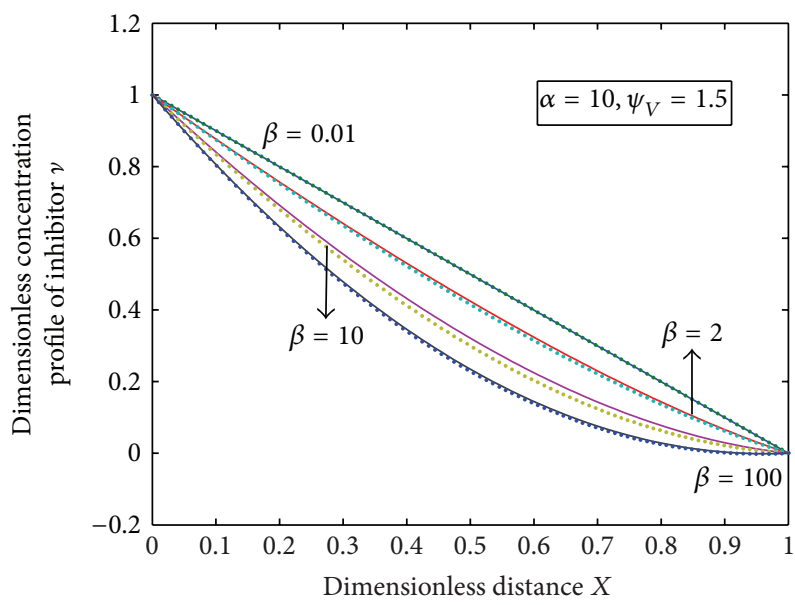

(e)

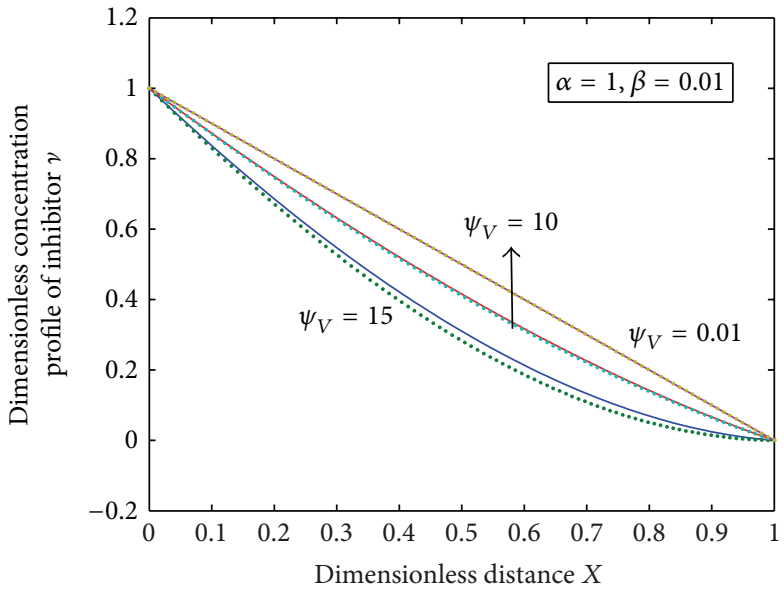

(b)

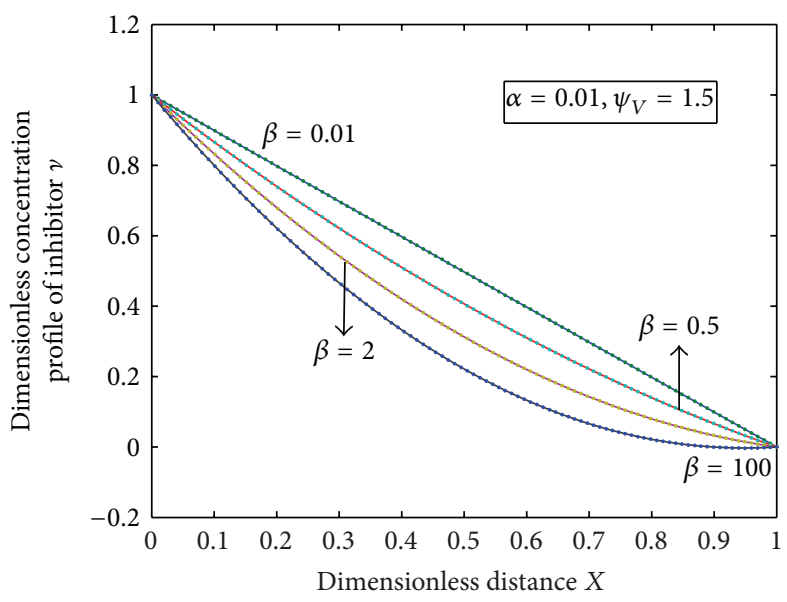

(d)

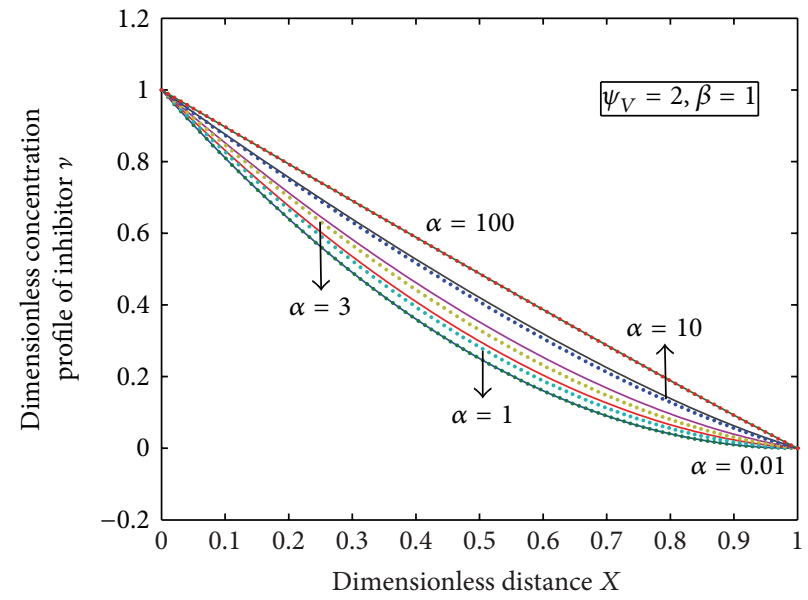

(f)

Figure 3: Plot of dimensionless inhibitor concentration $v$ versus dimensionless distance $X$ for various values of $\alpha, \beta$, and $\psi_{V}$. The key to the graph (solid line) represents our approximate solution presented in this study (see (17)) and (dotted line) represents the numerical solution. 


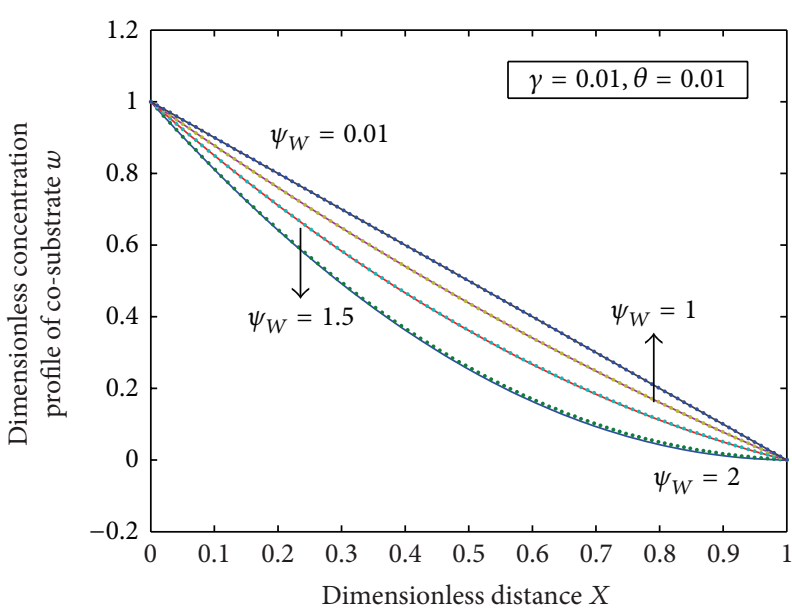

(a)

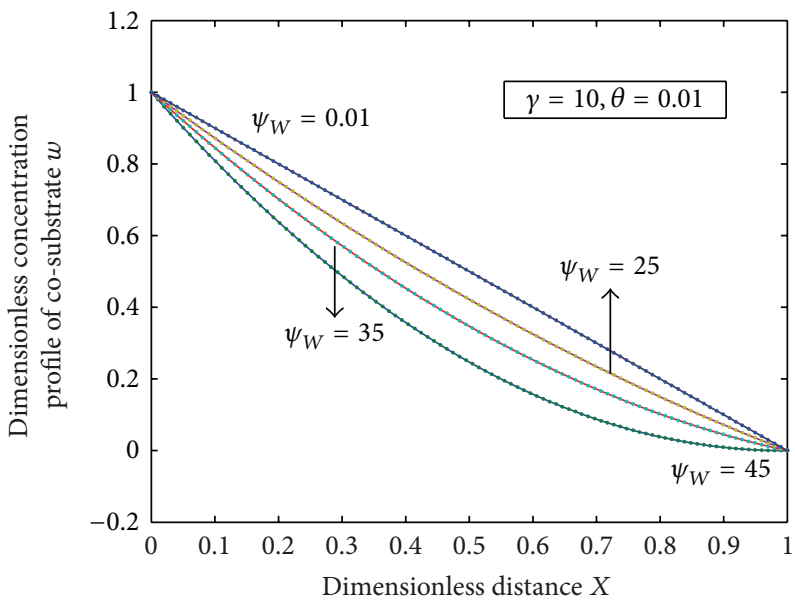

(c)

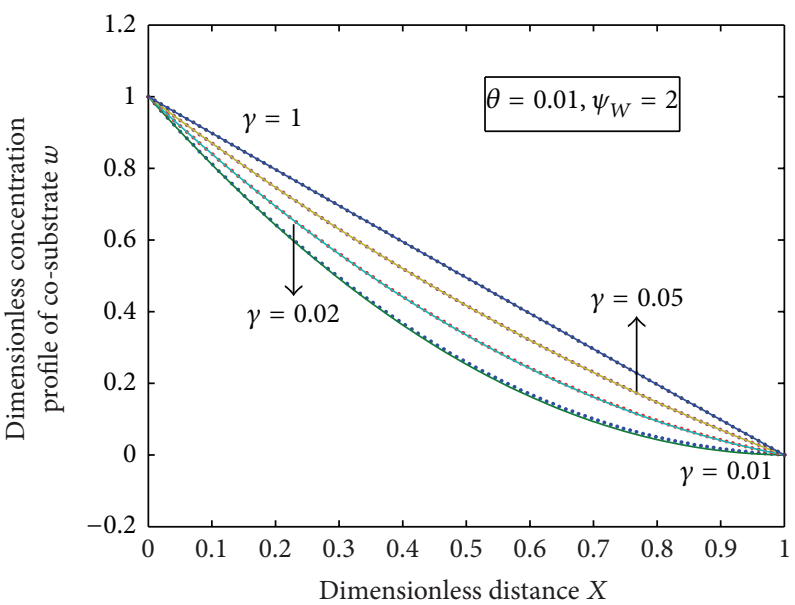

(e)

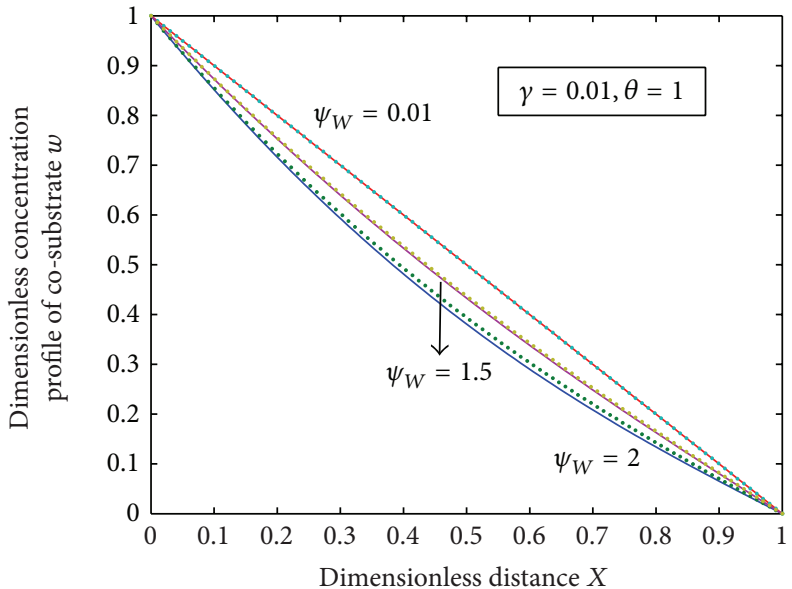

(b)

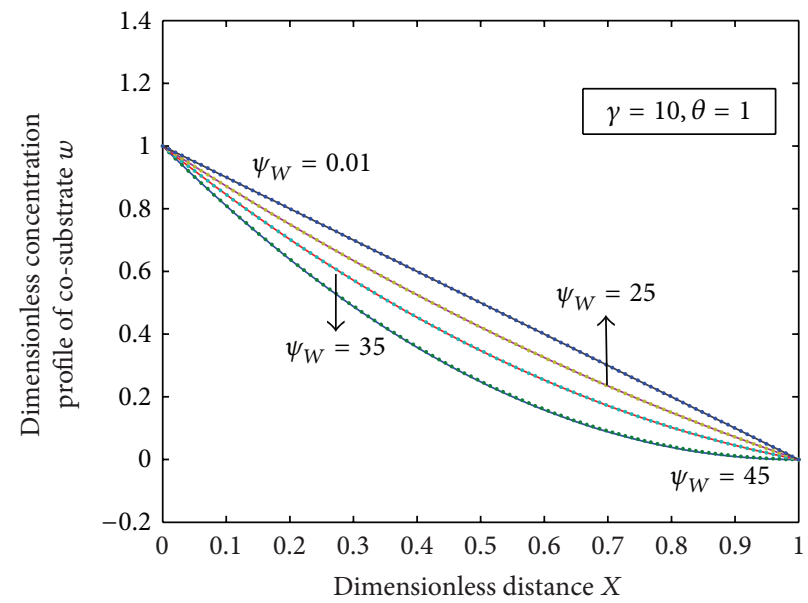

(d)

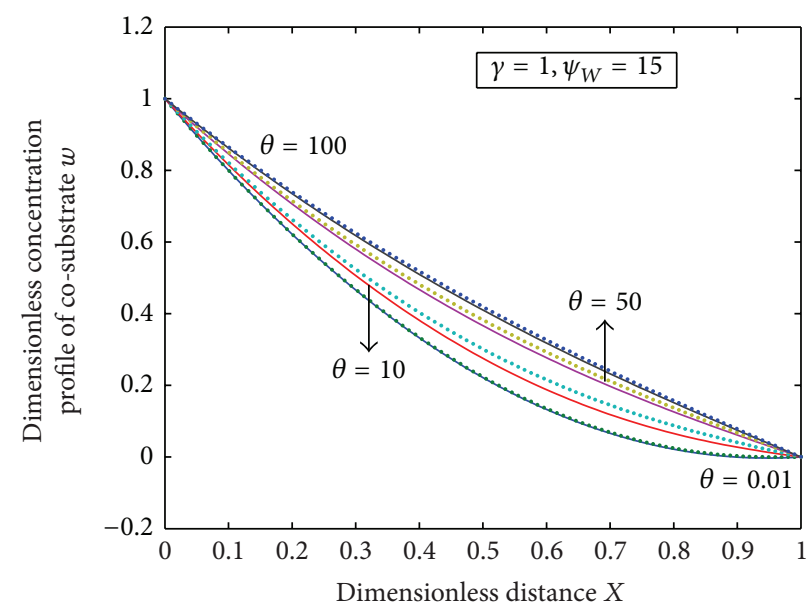

(f)

Figure 4: Plot of dimensionless co-substrate concentration $w$ versus dimensionless distance $X$ for various values of $\theta$, $\gamma$, and $\psi_{W}$. The key to the graph (solid line) represents our approximate solution presented in this study (see (18)) and (dotted line) represents the numerical solution. 


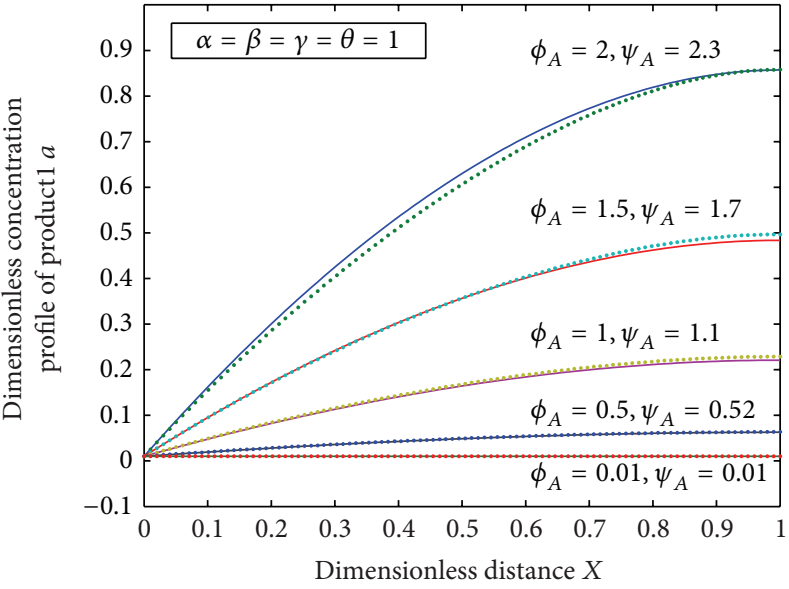

(a)

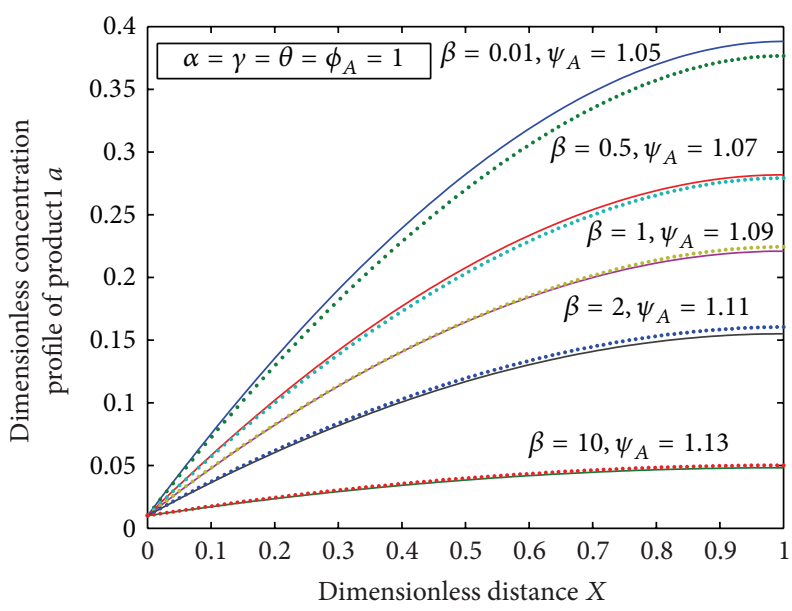

(c)

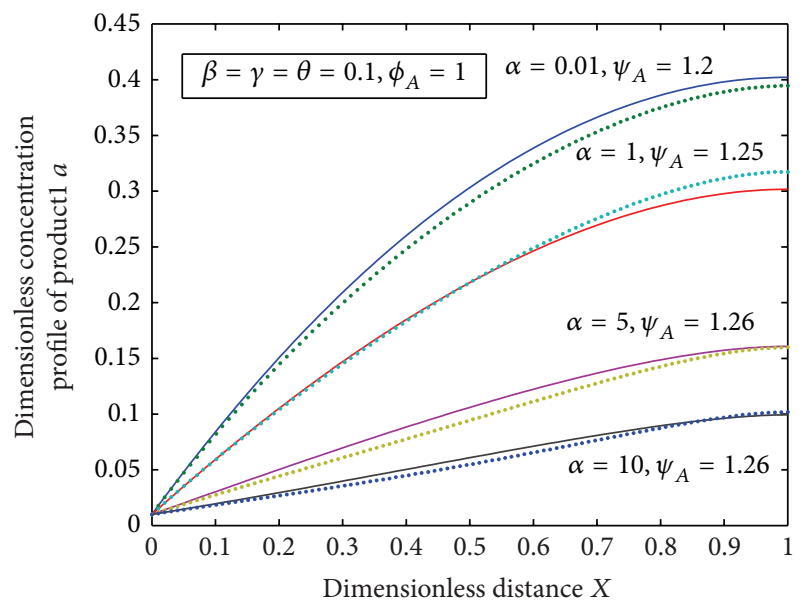

(e)

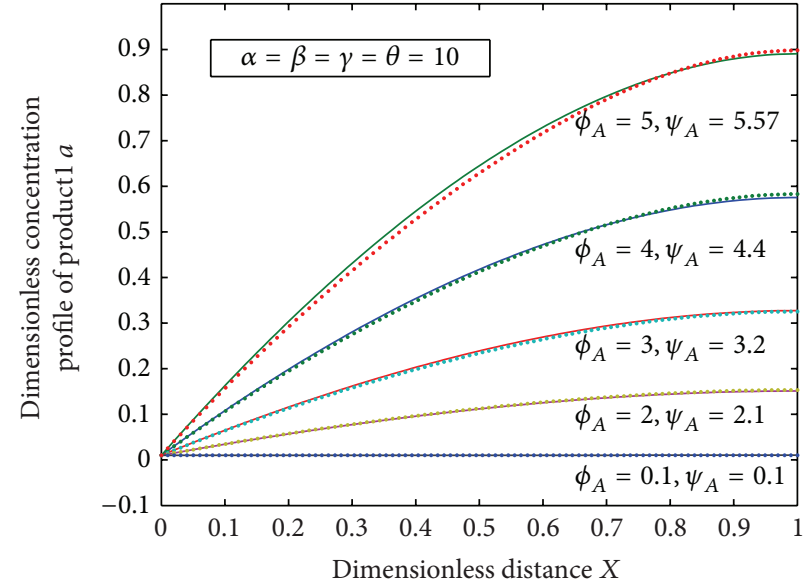

(b)

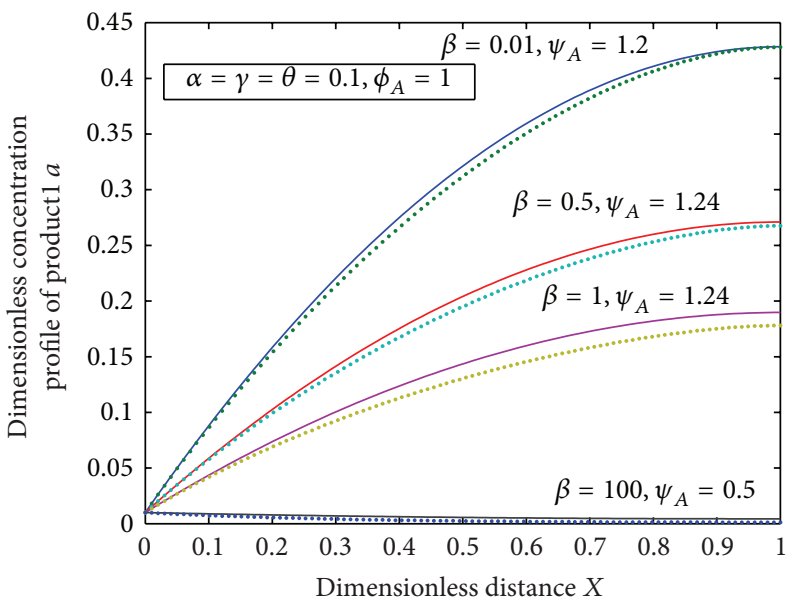

(d)

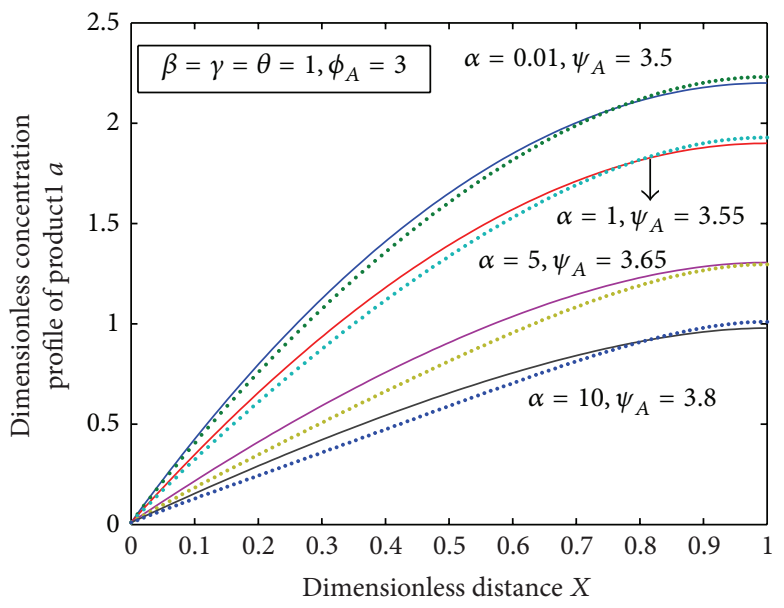

(f)

FIGURE 5: Plot of dimensionless product 1 concentration $a$ versus dimensionless distance $X$ for various values of $\alpha, \beta, \gamma, \theta, \phi_{A}$, and $\psi$. The key to the graph (solid line) represents our approximate solution presented in this study (see (19)) and (dotted line) represents the numerical solution. 


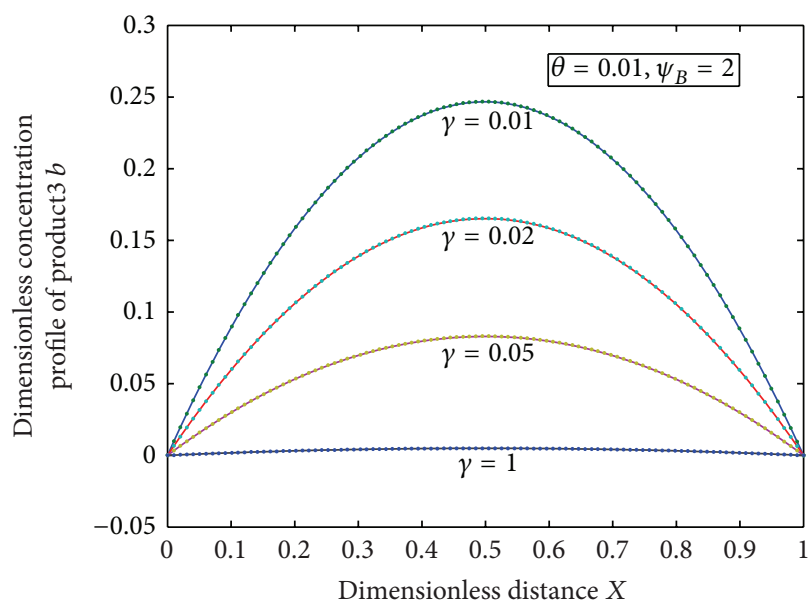

(a)

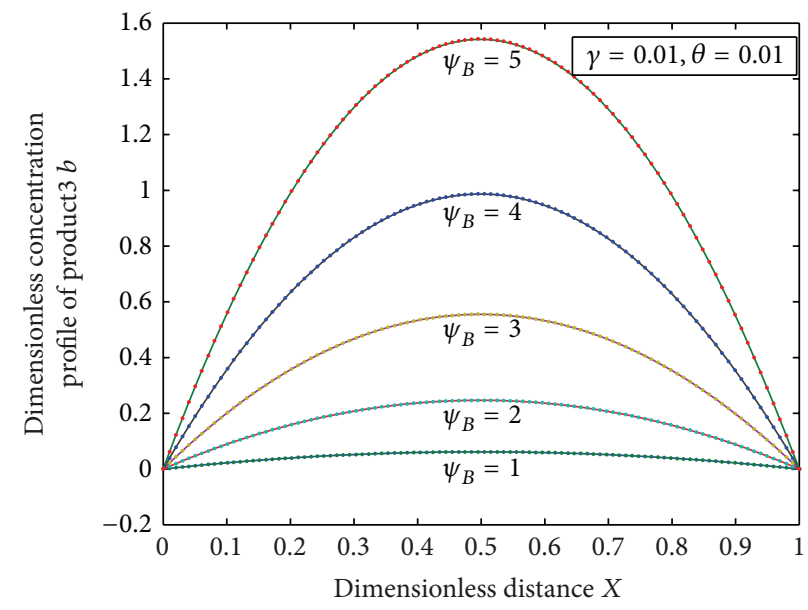

(c)

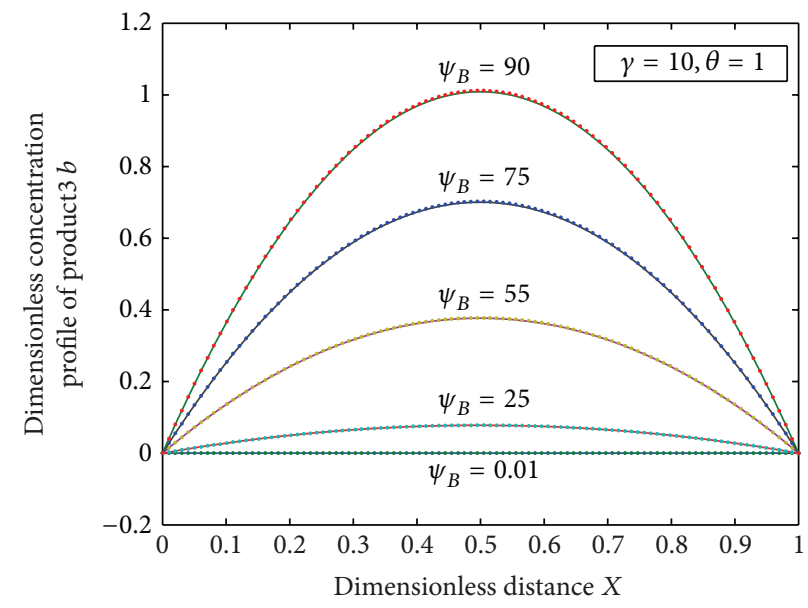

(e)

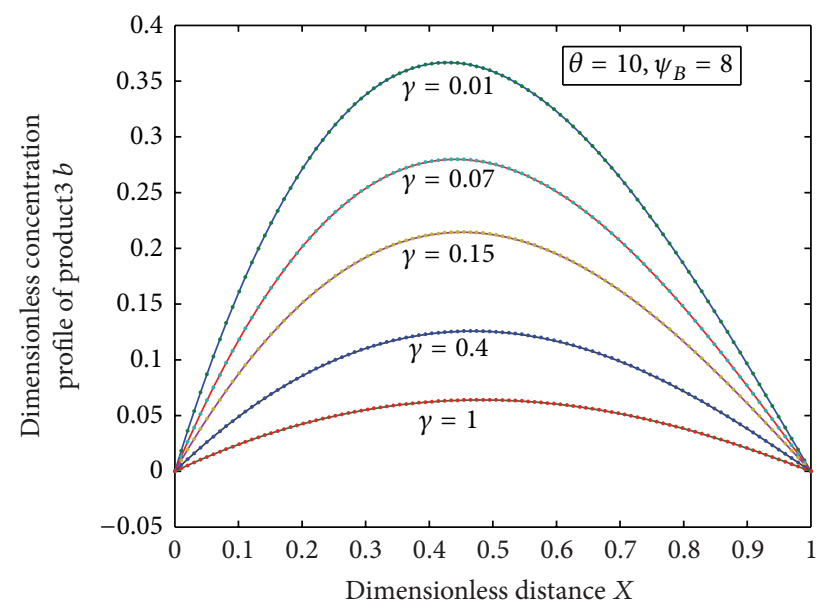

(b)

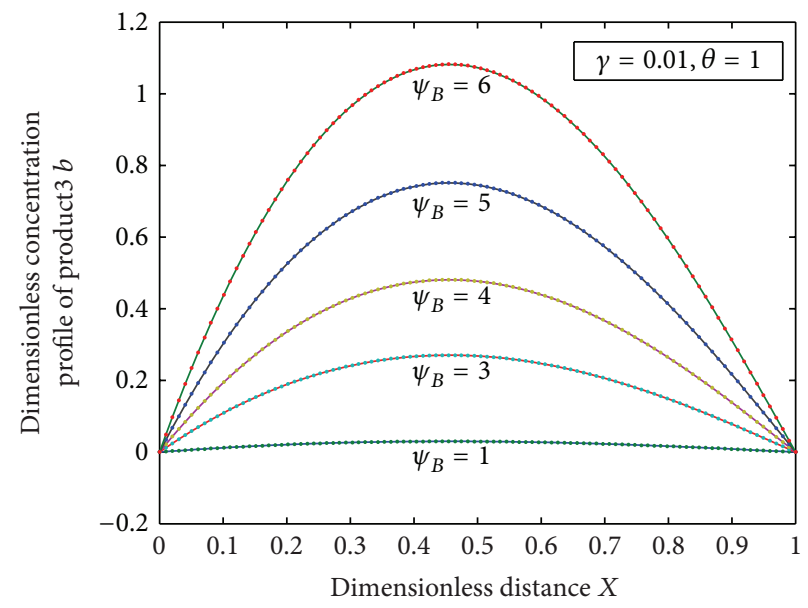

(d)

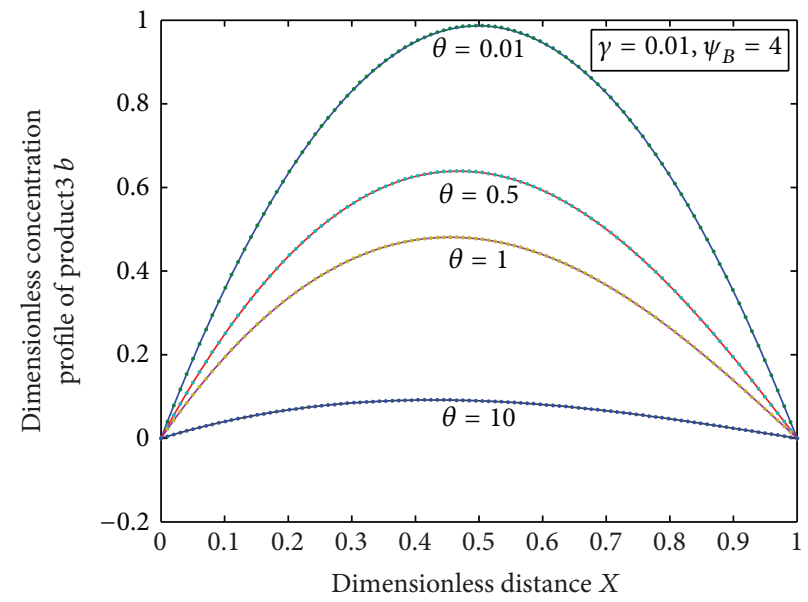

(f)

Figure 6: Plot of dimensionless product 3 concentration $b$ versus dimensionless distance $X$ for various values of $\theta, \gamma$, and $\psi_{B}$. The key to the graph (solid line) represents our approximate solution presented in this study (see (20)) and (dotted line) represents the numerical solution. 


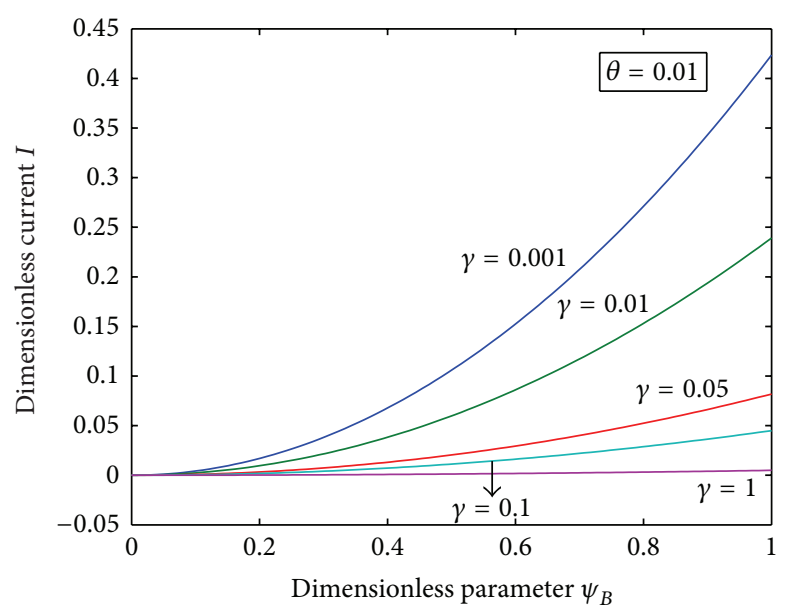

(a)

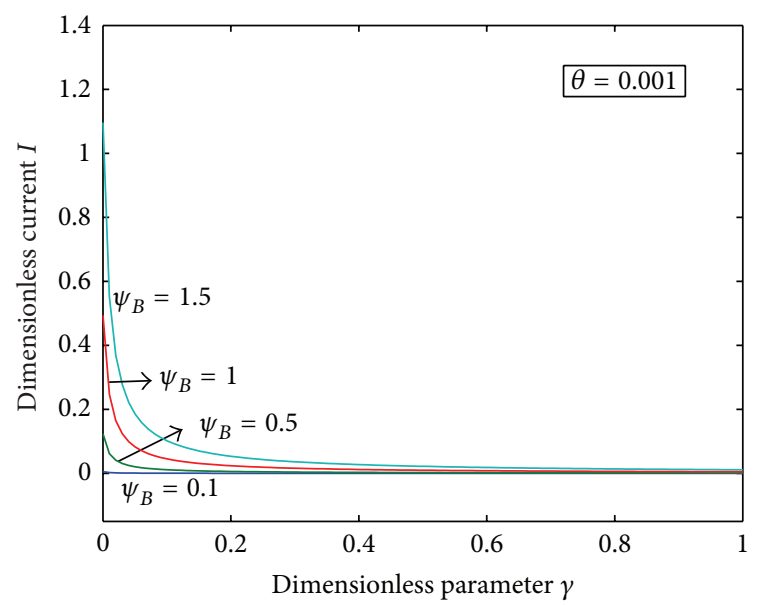

(c)

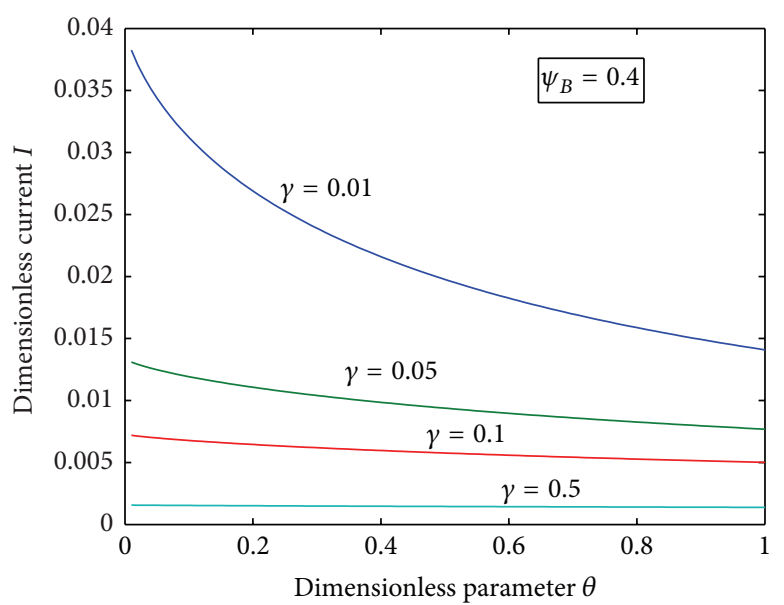

(e)

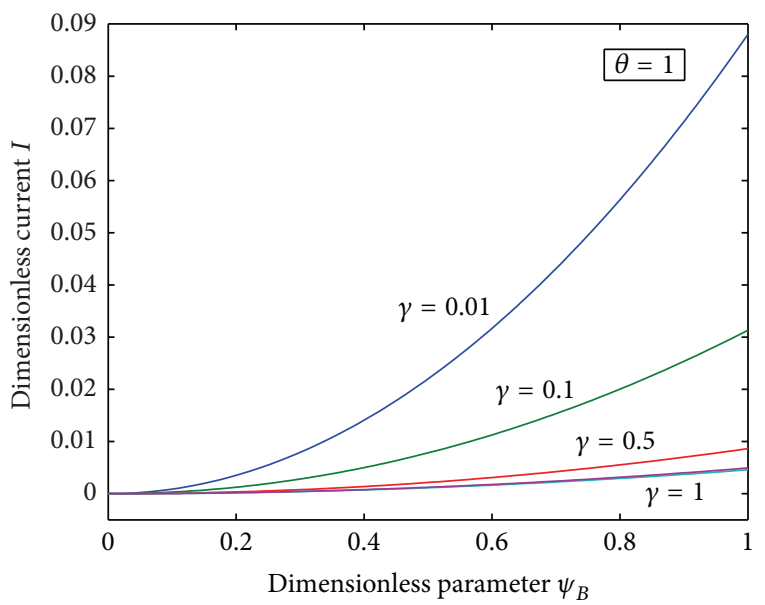

(b)

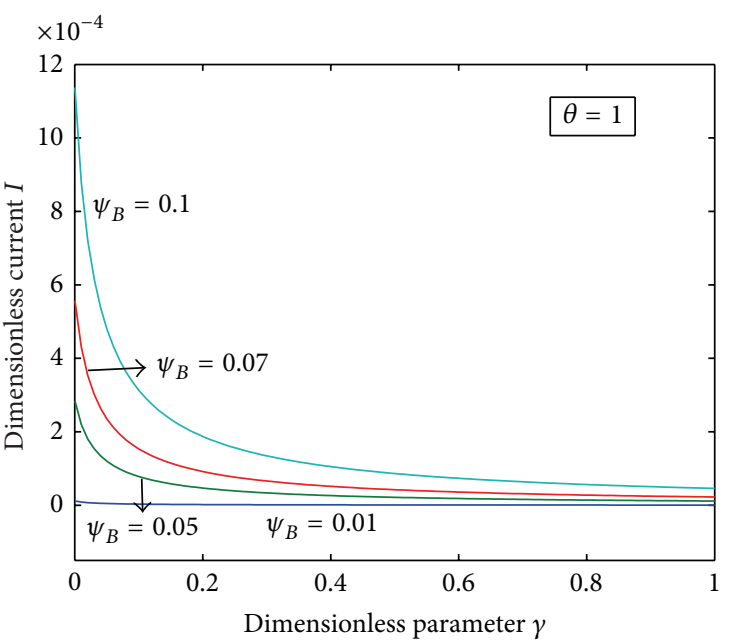

(d)

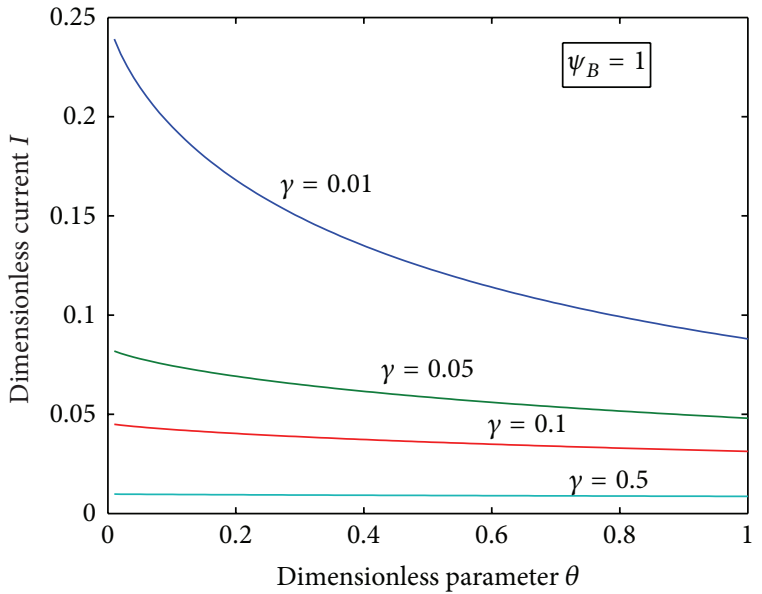

(f)

FIGURE 7: Generalized graph of the dimensionless current $I$ as a function of $\psi, \gamma, \theta$ calculated from (22). 
TABLE 1: Comparison of dimensionless concentration of glucose-6-phosphate $u$ with numerical simulation for various values of dimensionless distance $X$. (see Figure 2(a)).

\begin{tabular}{|c|c|c|c|c|c|c|c|c|c|c|c|c|}
\hline \multirow{3}{*}{$X$} & \multirow{2}{*}{\multicolumn{9}{|c|}{$\begin{aligned} & \alpha=0.01 \text { and } \phi_{U}=5 \\
\beta= & 50\end{aligned}$}} & \multirow{2}{*}{\multicolumn{3}{|c|}{$\beta=250$}} \\
\hline & & & & & & & & & & & & \\
\hline & $\begin{array}{c}\text { MADM } \\
\text { (this work) } \\
(16)\end{array}$ & NS* & $\begin{array}{c}\% \text { of } \\
\text { deviation }\end{array}$ & $\begin{array}{c}\text { MADM } \\
\text { (this work) } \\
(16)\end{array}$ & NS* & $\begin{array}{c}\% \text { of } \\
\text { deviation }\end{array}$ & $\begin{array}{c}\text { MADM } \\
\text { (this work) } \\
(16)\end{array}$ & $\mathrm{NS}^{*}$ & $\begin{array}{c}\% \text { of } \\
\text { deviation }\end{array}$ & $\begin{array}{c}\text { MADM } \\
\text { (this work) } \\
(16)\end{array}$ & $\mathrm{NS}^{*}$ & $\begin{array}{c}\% \text { of } \\
\text { deviation }\end{array}$ \\
\hline 0 & 1.000 & 1.000 & 0.000 & 1.000 & 1.000 & 0.000 & 1.000 & 1.000 & 0.000 & 1.000 & 1.000 & 0.000 \\
\hline 0.1 & 0.909 & 0.912 & 0.329 & 0.954 & 0.954 & 0.000 & 0.976 & 0.976 & 0.000 & 0.991 & 0.991 & 0.000 \\
\hline 0.2 & 0.828 & 0.833 & 0.600 & 0.912 & 0.912 & 0.000 & 0.955 & 0.955 & 0.000 & 0.982 & 0.982 & 0.000 \\
\hline 0.3 & 0.757 & 0.764 & 0.916 & 0.879 & 0.879 & 0.000 & 0.937 & 0.937 & 0.000 & 0.974 & 0.974 & 0.000 \\
\hline 0.4 & 0.701 & 0.709 & 1.128 & 0.847 & 0.847 & 0.000 & 0.922 & 0.922 & 0.000 & 0.968 & 0.968 & 0.000 \\
\hline 0.5 & 0.647 & 0.657 & 1.522 & 0.819 & 0.819 & 0.000 & 0.908 & 0.908 & 0.000 & 0.963 & 0.963 & 0.000 \\
\hline 0.6 & 0.603 & 0.615 & 1.951 & 0.798 & 0.798 & 0.000 & 0.897 & 0.897 & 0.000 & 0.958 & 0.958 & 0.000 \\
\hline 0.7 & 0.569 & 0.582 & 2.234 & 0.780 & 0.780 & 0.000 & 0.888 & 0.888 & 0.000 & 0.955 & 0.955 & 0.000 \\
\hline 0.8 & 0.545 & 0.559 & 2.504 & 0.768 & 0.768 & 0.000 & 0.882 & 0.882 & 0.000 & 0.952 & 0.952 & 0.000 \\
\hline 0.9 & 0.530 & 0.544 & 2.574 & 0.761 & 0.761 & 0.000 & 0.878 & 0.878 & 0.000 & 0.951 & 0.951 & 0.000 \\
\hline 1 & 0.525 & 0.539 & 2.597 & 0.758 & 0.758 & 0.000 & 0.877 & 0.877 & 0.000 & 0.950 & 0.950 & 0.000 \\
\hline \multicolumn{3}{|c|}{ Average $\%$ of deviation } & 1.487 & & & 0.000 & & & 0.000 & & & 0.000 \\
\hline
\end{tabular}

${ }^{*}$ NS: numerical simulation.

\section{Conclusions}

The system of nonlinear, steady-state reaction diffusion equations in multienzyme biosensor has been solved analytically using modified Adomian decomposition method. The accuracy of the approximate analytical solutions has been verified by comparison with numerical solutions. Satisfactory agreement is noted. This method is very effective, simple, and time saving. It also gives the better results. This method can be easily extensive to all kinds of nonlinear differential equations. Moreover, the behavior of inhibitor starting concentration over biosensor can be analyzed through the current expression.

\section{Appendices}

\section{A. Basic Concepts of the Modified Adomian Decomposition Method}

The modified Adomian decomposition method consists of decomposing the nonlinear differential equation

$$
F[x, y(x)]=0
$$

into two components

$$
L[y(x)]+N[y(x)]=0,
$$

where $L$ and $N$ are the linear and the nonlinear parts of $F$ respectively. The operator $L$ is assumed to be an invertible operator. Solving for $L(y)$ leads to

$$
L[y(x)]=-N[y(x)] .
$$

Applying the inverse operator $L^{-1}$ to both sides of (A.3) yields

$$
y(x)=-L^{-1}[N(y)]+\phi(x),
$$

where $\phi(x)$ is the function that satisfies the condition $L(\phi)=$ 0 . Now suppose that the solution $y$ can be represented as an infinite series of the form

$$
y(x)=\sum_{n=0}^{\infty} y_{n}
$$

The modified Adomian decomposition method assumes that the nonlinear term $N(y)$ can be written as an infinite series in terms of the Adomian polynomials $A_{n}$ :

$$
N(y)=\sum_{n=0}^{\infty} A_{n}
$$

where the Adomian polynomials $A_{n}$ of $N(y)$ are evaluated using the formula

$$
A_{n}(x)=\left.\frac{1}{n !} \frac{d^{n}}{d \lambda^{n}} N\left(\sum_{n=0}^{\infty} \lambda^{n} y_{n}\right)\right|_{\lambda=0}
$$

where $\lambda \in[0,1]$ is a hypothetical parameter.

Substituting (A.5) and (A.6) in (A.4) gives

$$
\sum_{n=0}^{\infty} y_{n}(x)=\phi(x)-L^{-1}\left(\sum_{n=0}^{\infty} A_{n}\right) .
$$

By equating the terms in the linear system of (A.8) one obtains the recurrence formula

$$
y_{0}(x)=\phi(x), \quad y_{n+1}(x)=-L^{-1}\left(A_{n}\right), \quad n \geq 0 .
$$

However, in practice all terms of the series (A.6) cannot be determined, and the solution is approximated by the truncated series $\sum_{n=0}^{N} y_{n}$. 


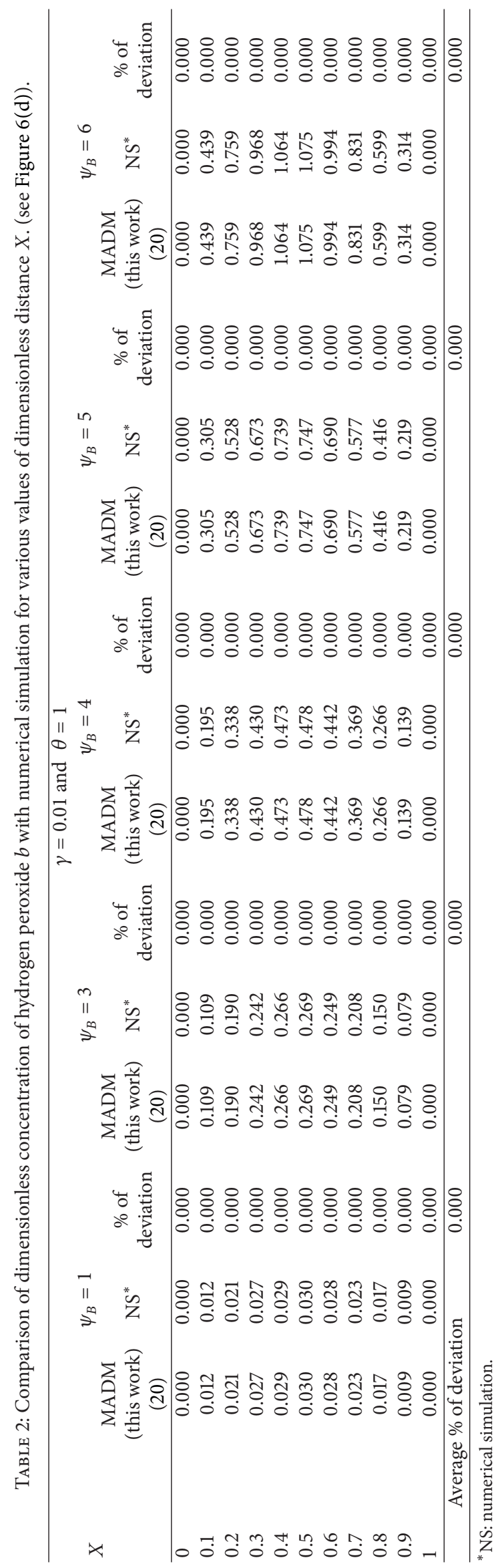




\section{B. Analytical Solutions for the Concentration Profiles of Five Reagents}

The analytical solution is necessary to predict the concentration profiles of glucose-6-phosphate, potassium dihydrogen phosphate, oxygen, glucose, and hydrogen peroxide solution of (9)-(13). Modified Adomian decomposition method is employed $[21,22]$ to construct approximate nonperturbative solutions of five reagents described by (9)-(13). In this appendix the expressions are derived for the concentrations of glucose-6-phosphate and potassium dihydrogen phosphate. The operator form is

$$
L\left[\begin{array}{l}
u(X) \\
v(X)
\end{array}\right]=N\left[\begin{array}{l}
u(X) \\
v(X)
\end{array}\right]=\left[\begin{array}{l}
\phi_{U}^{2} \\
\psi_{V}^{2}
\end{array}\right] \frac{u(X)}{1+\alpha v(X)+\beta u(X)},
$$

where $L=d^{2} / d X^{2}$. Applying the inverse operator $L^{-1}$ to both sides of (B.1) yields, in agreement with (A.4), (A.6), and (A.8)

$$
\begin{aligned}
{\left[\begin{array}{l}
u(X) \\
v(X)
\end{array}\right]=} & \sum_{n=0}^{\infty}\left[\begin{array}{l}
u_{n}(X) \\
v_{n}(X)
\end{array}\right] \\
= & {\left[\begin{array}{l}
A_{u} \\
A_{v}
\end{array}\right] X+\left[\begin{array}{l}
B_{u} \\
B_{v}
\end{array}\right] } \\
& +L^{-1}\left[\begin{array}{l}
\phi_{U}^{2} \\
\psi_{V}^{2}
\end{array}\right] \frac{u(X)}{1+\alpha v(X)+\beta u(X)}, \\
{\left[\begin{array}{c}
u(X) \\
v(X)
\end{array}\right]=} & {\left[\begin{array}{l}
A_{u} \\
A_{v}
\end{array}\right] X+\left[\begin{array}{l}
B_{u} \\
B_{v}
\end{array}\right]+L^{-1} \sum_{n=0}^{\infty}\left[\begin{array}{l}
A_{n, u} \\
A_{n, u}
\end{array}\right], }
\end{aligned}
$$

where $A_{u}, A_{v}, B_{u}$, and $B_{v}$ are the integration constant and the Adomian polynomial coefficients $A_{n, i}(X)(i=u, v)$ can be obtained using (A.7). By equating the terms of (B.3) and using the boundary conditions (14), we get

$$
\left[\begin{array}{l}
u_{0}(X) \\
v_{0}(X)
\end{array}\right]=\left[\begin{array}{l}
A_{u} \\
A_{v}
\end{array}\right] X+\left[\begin{array}{l}
B_{u} \\
B_{v}
\end{array}\right]=\left[\begin{array}{c}
1 \\
1-X
\end{array}\right]
$$

The first Adomian polynomial coefficients $A_{n, i}(X)(i=u, v)$ are

$$
\left[\begin{array}{c}
A_{0, u} \\
A_{0, v}
\end{array}\right]=\left[\begin{array}{l}
\phi_{U}^{2} \\
\psi_{V}^{2}
\end{array}\right] \frac{1}{1+\alpha(1-X)+\beta} .
$$

From the above equations we obtain $u_{1}(X), v_{1}(X)$ as follows:

$$
\left[\begin{array}{l}
u_{1}(X) \\
v_{1}(X)
\end{array}\right]=L^{-1}\left[\begin{array}{l}
A_{0, u} \\
A_{0, v}
\end{array}\right]=-\frac{1}{c^{2}}\left[\begin{array}{c}
\phi_{U}^{2}((N+1) c X-M) \\
\psi_{V}^{2}(N(c+d) X-M)
\end{array}\right],
$$

where

$$
\begin{gathered}
c=-\alpha, \quad d=1+\alpha+\beta, \quad N=\log \left(\frac{c+d}{d}\right), \\
M=(c X+d) \log \left(\frac{c X+d}{d}\right) .
\end{gathered}
$$

Adding (B.4) and (B.6) we obtain (16) and (17) in the text. Similarly we can find the analytical solution for (11)-(13).

\section{Matlab Code}

The Matlab program to find the numerical solution of (9)(13) is as shown in Algorithm 1.

\section{Notations}

S: Concentration of glucose-6-phosphate $\left(\mathrm{mol} / \mathrm{cm}^{3}\right)$

I: Concentration of potassium dihydrogen phosphate $\left(\mathrm{mol} / \mathrm{cm}^{3}\right)$

$C$ : Concentration of oxygen $\left(\mathrm{mol} / \mathrm{cm}^{3}\right)$

$P_{1}$ : Concentration of glucose $\left(\mathrm{mol} / \mathrm{cm}^{3}\right)$

$P_{2}$ : Concentration of inorganic phosphate, $\mathrm{HPO}_{4}^{2-}\left(\mathrm{mol} / \mathrm{cm}^{3}\right)$

$P_{3}$ : Concentration of hydrogen peroxide $\left(\mathrm{mol} / \mathrm{cm}^{3}\right)$

$P_{4}$ : Concentration of glucono delta-lactone, $\mathrm{C}_{6} \mathrm{H}_{10} \mathrm{O}_{6}\left(\mathrm{~mol} / \mathrm{cm}^{3}\right)$

$E_{1}$ : Acid phosphatase (AP)

$E_{2}$ : Glucose oxidase (GOD)

$V_{1}$ : Reaction velocity of acid phosphatase $\left(\mathrm{mol} / \mathrm{s} \mathrm{cm}^{3}\right.$ )

$V_{2}$ : Reaction velocity of glucose oxidase $\left(\mathrm{mol} / \mathrm{s} \mathrm{cm}^{3}\right.$ )

$K_{S}$ : Reaction constant for glucose-6-phosphate $\left(\mathrm{mol} / \mathrm{cm}^{3}\right)$

$K_{I}$ : Reaction constant for potassium dihydrogen phosphate $\left(\mathrm{mol} / \mathrm{cm}^{3}\right)$

$K_{C}$ : Reaction constant for oxygen $\left(\mathrm{mol} / \mathrm{cm}^{3}\right)$

$K_{P_{1}}$ : Reaction constant for glucose $\left(\mathrm{mol} / \mathrm{cm}^{3}\right)$

$D_{S}$ : Diffusion coefficient of glucose-6-phosphate $\left(\mathrm{cm}^{2} / \mathrm{s}\right)$

$D_{I}$ : Diffusion coefficient of potassium dihydrogen phosphate $\left(\mathrm{cm}^{2} / \mathrm{s}\right)$

$D_{C}$ : Diffusion coefficient of oxygen $\left(\mathrm{cm}^{2} / \mathrm{s}\right)$

$D_{P_{1}}$ : Diffusion coefficient of glucose $\left(\mathrm{cm}^{2} / \mathrm{s}\right)$

$D_{P_{3}}$ : Diffusion coefficient of hydrogen peroxide $\left(\mathrm{cm}^{2} / \mathrm{s}\right)$

$x:$ Distance $(\mathrm{cm})$

$S_{0}$ : Concentration of glucose-6-phosphate in bulk solution $\left(\mathrm{mol} / \mathrm{cm}^{3}\right)$

$I_{0}$ : Concentration of potassium dihydrogen phosphate in bulk solution $\left(\mathrm{mol} / \mathrm{cm}^{3}\right)$

$\mathrm{C}_{0}$ : Concentration of oxygen in bulk solution $\left(\mathrm{mol} / \mathrm{cm}^{3}\right)$

$d$ : Thickness of the membrane $(\mathrm{cm})$

$i$ : Output current at the electrode surface (ampere).

\section{Dimensionless Variables}

$u$ : Dimensionless concentration of glucose-6-phosphate

$v$ : Dimensionless concentration of potassium dihydrogen phosphate 
$w$ : Dimensionless concentration of oxygen

a: Dimensionless concentration of glucose

$b$ : Dimensionless concentration of hydrogen peroxide

$X$ : Dimensionless distance

I: Dimensionless current.

Greek Symbols

$\alpha: \quad$ Inhibitor inhibition modulus

(dimensionless)

$\beta$ : $\quad$ Substrate inhibition modulus

(dimensionless)

$\gamma, \theta: \quad$ Michaelis-Menten constants

(dimensionless)

$\phi_{U}: \quad$ Substrate Thiele modulus (dimensionless)

$\psi_{V}: \quad$ Inhibitor Thiele modulus (dimensionless)

$\psi_{W}: \quad$ Co-substrate Thiele modulus

(dimensionless)

$\phi_{A}, \psi_{A}$ : Product 1 Thiele modulus (dimensionless)

$\psi_{B}$ : $\quad$ Product 3 Thiele modulus

(dimensionless).

\section{Conflict of Interests}

The authors declare that there is no conflict of interests regarding the publication of this paper.

\section{Acknowledgments}

This work was supported by the Department of Science and Technology (DST) (no. SB/SI/PC-50/2012), New Delhi, India. The authors are thankful to The Principal, The Madura College, Madurai, and The Secretary, The Madura College Board, for their encouragement. The authors are grateful to the reviewer's valuable comments that improved the paper.

\section{References}

[1] A. Sadana and N. Sadana, Handbook of Biosensors and Biosensor Kinetics, Elsevier, New York, NY, USA, 2010.

[2] S. V. Dzyadevych, A. P. Soldatkin, Y. I. Korpan et al., "Biosensors based on enzyme field-effect transistors for determination of some substrates and inhibitors," Analytical and Bioanalytical Chemistry, vol. 377, no. 3, pp. 496-506, 2003.

[3] M. D. L. de Castro and M. C. Herrera, "Enzyme inhibitionbased biosensors and biosensing systems: questionable analytical devices," Biosensors and Bioelectronics, vol. 18, no. 2-3, pp. 279-294, 2003.

[4] A. Amine, H. Mohammadi, I. Bourais, and G. Palleschi, "Enzyme inhibition-based biosensors for food safety and environmental monitoring," Biosensors and Bioelectronics, vol. 21, no. 8, pp. 1405-1423, 2006.

[5] M. B. Gu, R. J. Mitchell, and B. C. Kim, "Whole-cell-based biosensors for environmental biomonitoring and application," Advances in Biochemical Engineering/Biotechnology, vol. 87, pp. 269-305, 2004.

[6] L. Campanella, F. Cubadda, M. P. Sammartino, and A. Saoncella, "An algal biosensor for the monitoring of water toxicity in estuarine environments,' Water Research, vol. 35, no. 1, pp. 6976, 2000.

[7] K. J. M. Sandström, J. Newman, A.-L. Sunesson, J.-O. Levin, and A. P. F. Turner, "Amperometric biosensor for formic acid in air," Sensors and Actuators B: Chemical, vol. 70, no. 1-3, pp. 182-187, 2000.

[8] Y. H. Lanyon, G. Marrazza, I. E. Tothill, and M. Mascini, "Benzene analysis in workplace air using an FIA-based bacterial biosensor," Biosensors and Bioelectronics, vol. 20, no. 10, pp. 2089-2096, 2005.

[9] F. N. Kok and V. Hasirci, "Determination of binary pesticide mixtures by an acetylcholinesterase- choline oxidase biosensor," Biosensors and Bioelectronics, vol. 19, no. 7, pp. 661-665, 2004.

[10] F. Mazzei, F. Botrè, and C. Botrè, "Acid phosphatase/glucose oxidase-based biosensors for the determination of pesticides," Analytica Chimica Acta, vol. 336, no. 1-3, pp. 67-75, 1996.

[11] G. Rahamathunissa and L. Rajendran, "Application of He's variational iteration method in nonlinear boundary value problems in enzyme-substrate reaction diffusion processes: part 1 . The steady-state amperometric response," Journal of Mathematical Chemistry, vol. 44, no. 3, pp. 849-861, 2008.

[12] G. Rahamathunissa, P. Manisankar, L. Rajendran, and K. Venugopal, "Modeling of nonlinear boundary value problems in enzyme-catalyzed reaction diffusion processes," Journal of Mathematical Chemistry, vol. 49, no. 2, pp. 457-474, 2011.

[13] X. Zhang, H. Ju, and J. Wang, Eds., Electrochemical Sensors, Biosensors and their Biomedical Applications, Academic Press, New York, NY, USA, 2007.

[14] J.-Y. Yoon, Introduction to Biosensors: From Electric Circuits to Immunosensors, Springer, 2013.

[15] R. Baronas, F. Ivanauskas, and J. Kulys, Mathematical Modeling of Biosensors: An Introduction for Chemists and Mathematicians, Springer Series on Chemical Sensors and Biosensors, Springer, Dordrecht, The Netherlands, 2010.

[16] L. Rajendran, Chemical Sensors: Simulation and Modeling Volume 5: Electrochemical Sensors, Edited by: G. Korotcenkov, Momentum press, New York, NY, USA, 2013.

[17] V. Rangelova, A. Pandelova, and N. Stoiyanov, "Inhibitor multienzyme biosensor system in dynamic mode-phosphate measurement," Annals of Faculty Engineering HunedoaraInternational Journal of Engineering, vol. 9, no. 2, pp. 83-86, 2011.

[18] G. Adomian, "Convergent series solution of nonlinear equations," Journal of Computational and Applied Mathematics, vol. 11, no. 2, pp. 225-230, 1984.

[19] G. Adomian, "A review of the decomposition method and some recent results for nonlinear equations," Mathematical and Computer Modelling, vol. 13, no. 7, pp. 17-43, 1990.

[20] Y. Q. Hasan and L. M. Zhu, "Modified adomian decomposition method for singular initial value problems in the second-order ordinary differential equations," Surveys in Mathematics and Its Applications, vol. 3, pp. 183-193, 2008.

[21] M. M. Hosseini, "Adomian decomposition method with Chebyshev polynomials," Applied Mathematics and Computation, vol. 175, no. 2, pp. 1685-1693, 2006.

[22] A.-M. Wazwaz, "A new algorithm for calculating Adomian polynomials for nonlinear operators," Applied Mathematics and Computation, vol. 111, no. 1, pp. 53-69, 2000. 


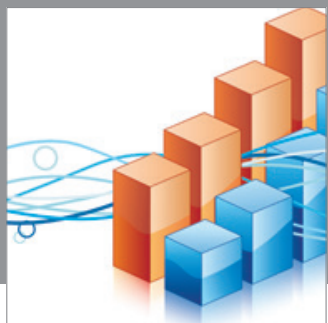

Advances in

Operations Research

mansans

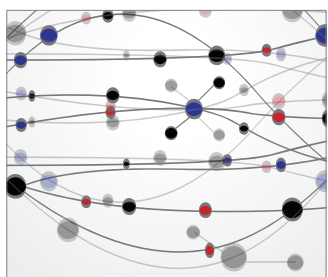

The Scientific World Journal
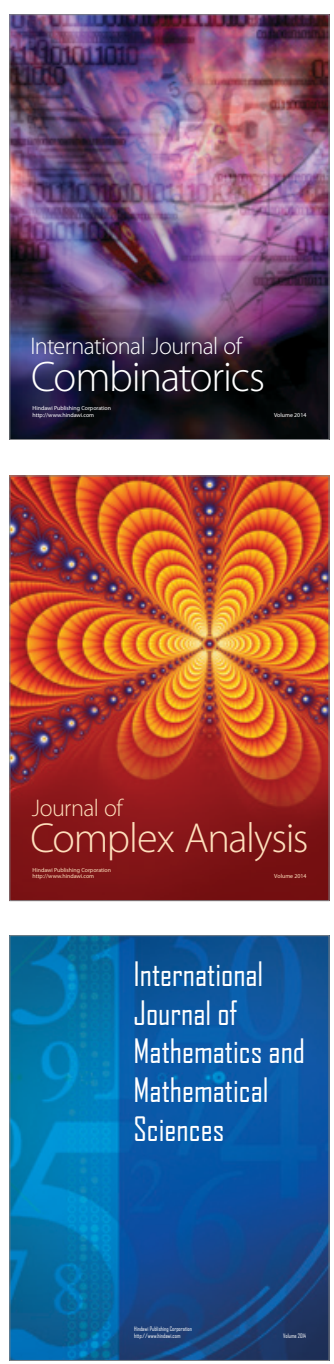
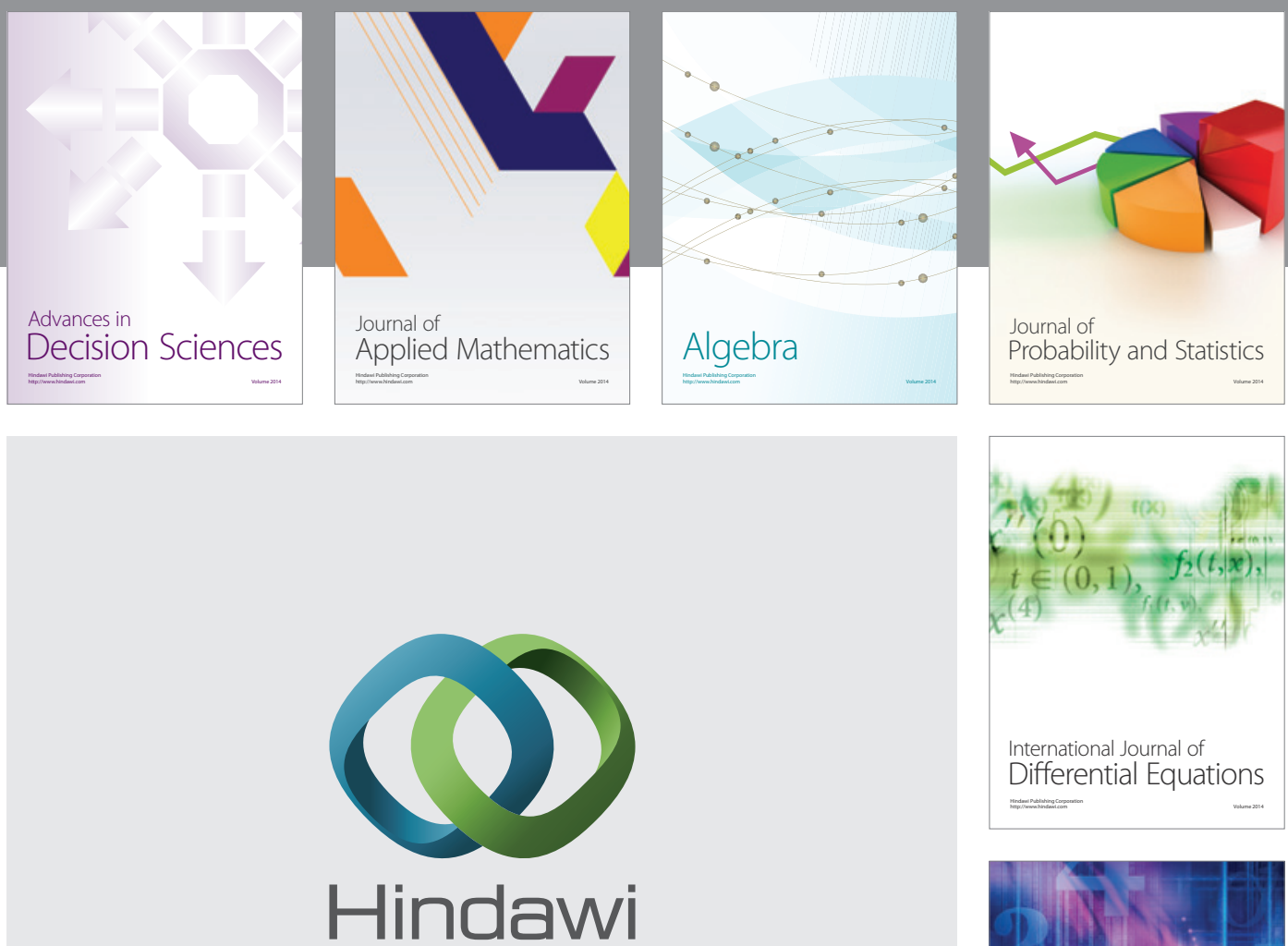

Submit your manuscripts at http://www.hindawi.com
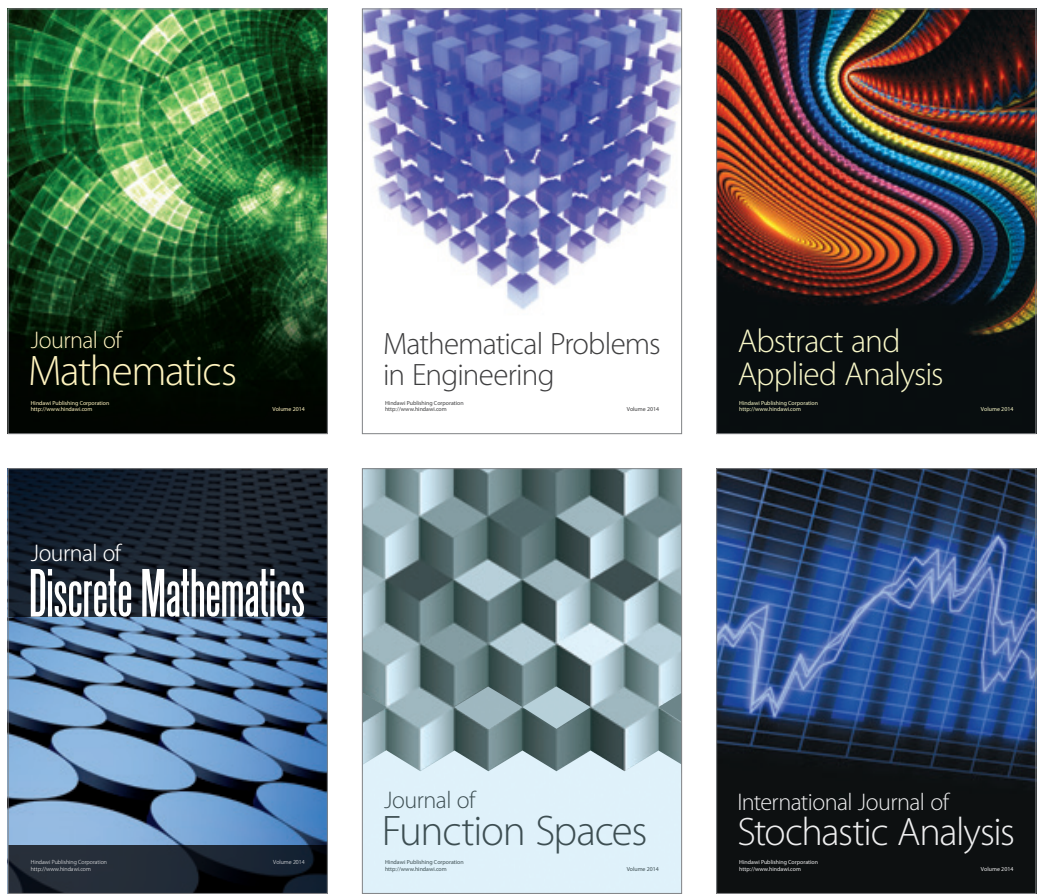

Journal of

Function Spaces

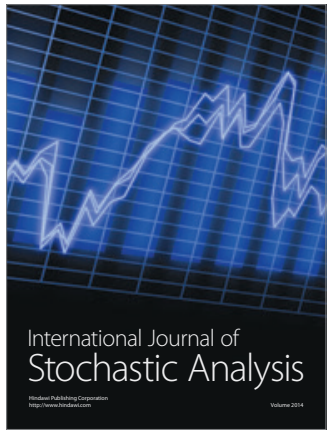

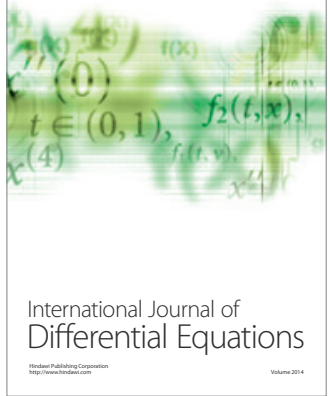
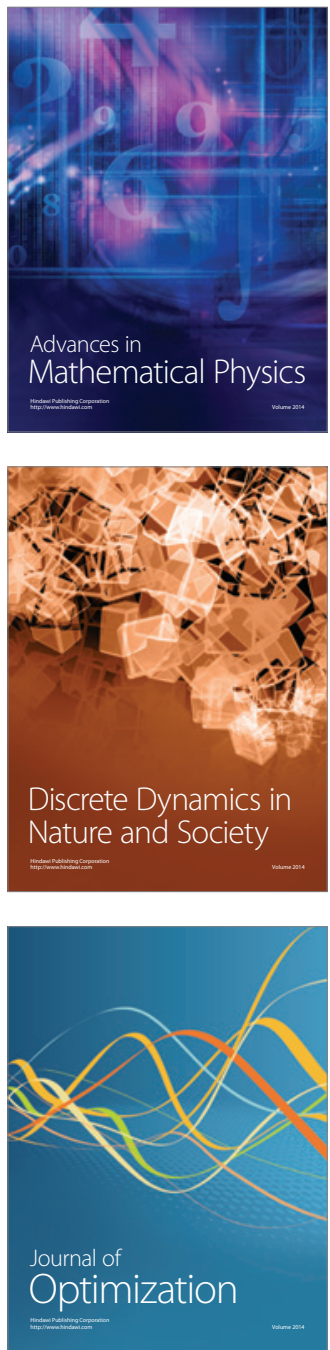